MATHEMATICS OF COMPUTATION

Volume 74, Number 249, Pages 153-176

S 0025-5718(04)01635-7

Article electronically published on March 18, 2004

\title{
ABSORBING BOUNDARY CONDITIONS FOR THE WAVE EQUATION AND PARALLEL COMPUTING
}

\author{
MARTIN J. GANDER AND LAURENCE HALPERN
}

\begin{abstract}
Absorbing boundary conditions have been developed for various types of problems to truncate infinite domains in order to perform computations. But absorbing boundary conditions have a second, recent and important application: parallel computing. We show that absorbing boundary conditions are essential for a good performance of the Schwarz waveform relaxation algorithm applied to the wave equation. In turn this application gives the idea of introducing a layer close to the truncation boundary which leads to a new way of optimizing absorbing boundary conditions for truncating domains. We optimize the conditions in the case of straight boundaries and illustrate our analysis with numerical experiments both for truncating domains and the Schwarz waveform relaxation algorithm.
\end{abstract}

\section{INTRODUCTION}

Absorbing boundary conditions have been introduced in the seminal papers by Engquist and Majda [15] and Bayliss and Turkel [2] to truncate infinite domains in order to perform computations of wave propagation phenomena in acoustics and fluid dynamics. The idea is to impose a boundary condition at the truncation boundary which is as invisible as possible for the waves, so that they can exit the computational domain as if there were no boundary. This has the effect that the solution computed on the truncated domain is close to the solution on the infinite domain. The main difficulty is that exact absorbing boundary conditions, conditions that absorb all the information, are almost always nonlocal and thus are expensive to use. The challenge is to find approximations which are computationally inexpensive yet effective for the solution sought in the computation. In the strategy developed by Engquist and Majda, a perfectly absorbing boundary condition is obtained through the theory of pseudo differential operators. This condition is given by the Dirichlet to Neumann map. Then approximations are sought with respect to the frequency and the angle of incidence on the boundary, when curved boundaries are used. Such approximations are either Taylor or Padé approximations of the symbol, or optimized approximations (see 44]). A tremendous activity has followed to develop alternative strategies in various domains; for reviews see [30, [43. For diffusive problems, absorbing boundary conditions have been developed in 29] and extensive studies of the behavior of such conditions both at the continuous and discrete level followed; see for example [28, [14].

Received by the editor September 10, 2002 and, in revised form, May 12, 2003.

2000 Mathematics Subject Classification. Primary 65M55, 35L20.

(C)2004 American Mathematical Society 
In parallel computing, the importance of absorbing boundary conditions was only realized quite late. For Helmholtz problems, where the classical domain decomposition methods failed because of the propagating waves, classical radiation conditions were introduced for domain decomposition in [12]. This approach was further developed and analyzed in [3], [7, [11] and 35. Higher order radiation conditions were then proposed for domain decomposition methods for the Helmholtz equation in [13] and [10] and a first attempt to optimize the performance of the algorithm can be found in [9]. This led to the new class of optimized Schwarz methods which were analyzed in depth for the Helmholtz equation in 24] and [19]. A different approach using perfectly matched layers can be found in [42.

For steady convection diffusion problems, a domain decomposition method using absorbing boundary conditions was first proposed in [8]. The optimal transmission conditions were further analyzed in [38 and optimized second order transmission conditions were introduced in [31] and further analyzed in [33] and 32]. The term optimized Schwarz methods first appeared in [23], where the effectiveness of those methods over the classical Schwarz method is shown for several types of problems.

The classical approach in domain decomposition for evolution problems is to first discretize the problem uniformly in time using an implicit discretization and then to apply at each time step one of the domain decomposition methods above; see for example [36, 5] and [6] for parabolic problems or [1] and [46] for hyperbolic problems. The main disadvantage of this classical approach is that one is forced to use the same time step in all subdomains and thus loses one of the main features of domain decomposition, namely treating subdomains numerically differently. A second disadvantage is that one needs to exchange information at each time step. Schwarz waveform relaxation is a remedy for both problems. In these methods the computational domain is partitioned into subdomains, as in the classical Schwarz method. However on subdomains, time dependent problems are solved in the iteration and thus the algorithm is also of waveform relaxation type. These methods have been introduced for diffusive evolution problems in [16] and independently in [27] and were further analyzed in [26], [17] and 25]. For nonlinear equations, see [18]. But as in the steady state case, the convergence of these algorithms can be greatly enhanced using absorbing transmission conditions [22]. For hyperbolic problems Schwarz waveform relaxation was first analyzed in [4] for Dirichlet transmission conditions and with optimal transmission conditions for the one dimensional wave equation in [20] and [21].

In this paper we study absorbing boundary conditions and Schwarz waveform relaxation for the linear wave equation in $d$ dimensions. We first analyze in Section 2 the classical overlapping Schwarz waveform relaxation algorithm which uses Dirichlet transmission conditions and we prove a convergence result for general overlapping decompositions unusual both for Schwarz methods and waveform relaxation: the algorithm converges on bounded time intervals in a finite number of steps. In Section 3 we introduce absorbing boundary conditions for the wave equation and show that they are nonlocal in general and thus expensive to use in numerical computations. We therefore introduce a general approximation and study the reflection coefficient resulting from the approximation. In Section 4 we show what influence the absorbing boundary conditions have when used as transmission conditions in the Schwarz waveform relaxation algorithm. Convergence is again obtained in a finite number of steps, but for a very different reason than in 
the result in Section[2. Convergence is obtained for all time, and even without overlap. In Section 5 we choose a particular rational approximation for the absorbing boundary conditions which treats propagating waves effectively, while evanescent waves are treated by a small layer. We optimize the conditions for truncating computational domains and for the performance of the Schwarz waveform relaxation algorithm, where we build on both convergence results obtained before to get the most effective algorithm. The section finishes with asymptotic convergence results as the mesh parameter goes to zero. We finally show in Section 6 numerical experiments both for truncated domains and for the optimized Schwarz waveform relaxation algorithm.

\section{Classical Schwarz Waveform Relaxation}

We are interested in solving the wave equation in $d$ dimensions on a parallel computer with slow communication links. We consider the equation for $u(\mathbf{x}, t)$ on a bounded domain $\Omega \subset \mathbb{R}^{d}$ with a smooth boundary $\Gamma$,

$$
\begin{array}{rlrl}
\square_{c} u:=\frac{1}{c^{2}} \frac{\partial^{2} u}{\partial t^{2}}-\Delta u & =f \quad & \text { in } \Omega \times(0, T), \\
u & =g \quad & \text { on } \Gamma \times(0, T), \\
u(\cdot, 0) & =u_{0} \quad \text { in } \Omega, \\
\frac{\partial u}{\partial t}(\cdot, 0) & =u_{0}^{\prime} & \text { in } \Omega .
\end{array}
$$

We assume that $u_{0} \in H^{1}(\Omega), u_{0}^{\prime} \in L^{2}(\Omega), f \in L^{2}\left(0, T ; L^{2}(\Omega)\right), g \in L^{2}\left(0, T ; H^{\frac{1}{2}}(\Gamma)\right)$ and that the wave speed satisfies $0<\underline{c} \leq c(\mathbf{x}) \leq \bar{c}$ a.e. in $\Omega$. Then there exists a unique solution $u \in L^{2}\left(0, T ; H^{1}(\Omega)\right)$ with $\frac{\partial u}{\partial t} \in L^{2}\left(0, T ; L^{2}(\Omega)\right)$; see [34.

To distribute the computation, we partition the domain $\Omega$ into overlapping subdomains. Such a partition can be obtained by first partitioning $\Omega$ into $J$ nonoverlapping subdomains $\widetilde{\Omega}_{j}$, where we denote the boundaries of the subdomain $\widetilde{\Omega}_{j}$ interior to the domain $\Omega$ by $\widetilde{\Gamma}_{j}$ and the part shared with the original boundary $\Gamma$ by $\widetilde{\Gamma}_{j}^{0}$. Then we construct an overlapping decomposition $\Omega_{j}$ with overlap parameter $\delta$ by enlarging each $\widetilde{\Omega}_{j}$ so that the boundaries of the new subdomains $\Gamma_{j}$ interior to $\Omega$ are at least at a distance $\delta+\epsilon$ away from $\widetilde{\Gamma}_{j}$; see Figure 1 for an example in two dimensions. As before we denote the part of the boundary subdomain $\Omega_{j}$ shares with the original boundary $\Gamma$ by $\Gamma_{j}^{0}$.

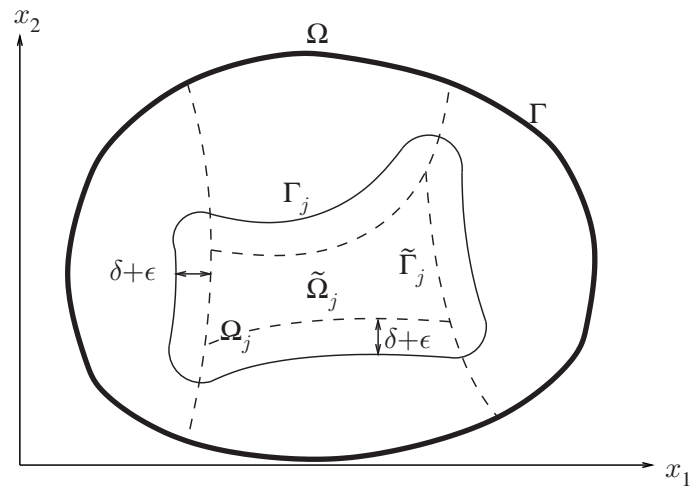

FIGURE 1. The construction of an overlapping decomposition in two dimensions. 
We further introduce for technical reasons a partition of unity $\chi_{j} \in C^{\infty}$ associated with the nonoverlapping subdomains $\widetilde{\Omega}_{j}$ such that $\chi_{j}=1$ in $\widetilde{\Omega}_{j}$ and $\chi_{j}=0$ outside, except for a small transition layer centered around the interior boundary $\widetilde{\Gamma}_{j}$ of width $2 \epsilon>0$ where $\chi_{j}$ decays smoothly to zero.

To solve the wave equation (2.1), the classical Schwarz waveform relaxation algorithm starts with an initial guess $u^{0} \in L^{2}\left(0, T ; H^{1}(\Omega)\right)$ and constructs successive approximations $u^{n} \in L^{2}\left(0, T ; H^{1}(\Omega)\right)$ by solving subdomain problems in the subdomains $\Omega_{j}$ only. Once all the subdomain solutions $u_{j}^{n} \in L^{2}\left(0, T ; H^{1}\left(\Omega_{j}\right)\right)$ are obtained, the new global iterate $u^{n}$ is defined using the partition of unity, $u^{n}:=\sum_{j=1}^{J} \chi_{j} u_{j}^{n}$, and hence $u^{n} \in L^{2}\left(0, T ; H^{1}(\Omega)\right)$. The classical Schwarz waveform relaxation algorithm is thus given by

$$
\begin{aligned}
& \square_{c} u_{j}^{n}=f \quad \text { in } \Omega_{j} \times(0, T) \text {, } \\
& u_{j}^{n}=u^{n-1} \text { on } \Gamma_{j} \times(0, T) \text {, } \\
& u_{j}^{n}=g \quad \text { on } \Gamma_{j}^{0} \times(0, T), \\
& u_{j}^{n}(\cdot, 0)=u_{0} \quad \text { in } \Omega_{j} \text {, } \\
& \frac{\partial u_{j}^{n}}{\partial t}(\cdot, 0)=u_{0}^{\prime} \quad \text { in } \Omega_{j}, \\
& u^{n}=\sum_{j=1}^{J} \chi_{j} u_{j}^{n} .
\end{aligned}
$$

The algorithm (2.2) corresponds to an additive Schwarz or Jacobi iteration which can be done in parallel. One can also consider a multiplicative Schwarz or Gauss Seidel iteration which would need a special coloring of subdomains to remain a parallel algorithm. If subdomains with the same color do not touch each other, then subdomains of the same color can be solved in parallel using the boundary values coming from subdomains with different colors. We focus in the analysis on the additive version; the results for the multiplicative versions can be obtained similarly.

Theorem 2.1 (Convergence in a finite number of steps). For given initial conditions $u_{0} \in H^{1}(\Omega), u_{0}^{\prime} \in L^{2}(\Omega)$, forcing function $f \in L^{2}\left(0, T ; L^{2}(\Omega)\right)$, boundary condition $g \in L^{2}\left(0, T ; H^{\frac{1}{2}}(\Gamma)\right)$ and initial guess $u^{0} \in L^{2}\left(0, T ; H^{1}(\Omega)\right)$, the classical overlapping Schwarz waveform relaxation algorithm (2.2) for the wave equation has converged in $L^{2}\left(0, T ; H^{1}(\Omega)\right)$ as soon as the number of iterations $n$ satisfies

$$
n>\frac{T \bar{c}}{\delta}, \quad \bar{c}:=\sup _{\mathbf{x} \in \Omega} c(\mathbf{x}) .
$$

Proof. The proof follows from the finite speed of propagation in the wave equation. By linearity it suffices to consider the homogeneous problem, $f=g=u_{0}=u_{0}^{\prime}=0$ in (2.2) which corresponds to the error equations, and to analyze convergence to zero. Without loss of generality, we can look at one subdomain only, say $\Omega_{j}$. At the first iteration the conditions imposed on its boundary are in general nonzero, since the solution is not known, so the error obtained in $\Omega_{j}$ will be nonzero, except in a cone within $\Omega_{j}$ where the initial conditions only are determining the solution. Since the initial conditions are zero (the solution is known there, and hence the error is zero), the error vanishes in this cone. This result holds for all subdomains, and since the solutions obtained in each subdomain are only used with the partition of unity within the smaller subdomains $\widetilde{\Omega}_{j}$ enlarged by $\epsilon$, the error is zero everywhere as long as $t<\frac{\delta}{\bar{c}}$, because this is the time the error needs to travel from the boundary of $\Omega_{j}$ across the layer of width $\delta$ to be reused in the next iteration. By induction after $n$ iterations the error will be uniformly zero if $t<n \frac{\delta}{\bar{c}}$. Now using that we are interested only in computations for $t \in[0, T]$, the result follows. 
This result is different from the classical results known for Schwarz methods and waveform relaxation. Schwarz methods for elliptic problems converge linearly [41] and waveform relaxation algorithms for evolution problems converge superlinearly [37. In the classical overlapping Schwarz waveform relaxation algorithm for the wave equation, reflected waves reentering the subdomain instead of leaving it are leading to the convergence result given in Theorem 2.1. Can convergence be improved if the transmission conditions in the algorithm are changed? We give the answer to this question by first analyzing the behavior of the solution at the interface between subdomains and then analyzing the algorithm with new transmission conditions in the next two sections.

\section{ABSORBING BOUNDARY CONDITIONS}

3.1. Exact absorbing boundary conditions. We consider the wave equation (2.1) on the bounded domain $\Omega$ in $\mathbb{R}^{d}$ as shown for a two dimensional example in Figure 2 on the left. If the domain is partitioned into two nonoverlapping subdomains $\widetilde{\Omega}_{1}$ and $\widetilde{\Omega}_{2}$ with the interface $\widetilde{\Gamma}$, as shown in Figure 2 on the right for a two dimensional example, then the original problem (2.1) is equivalent to the coupled problem

$$
\begin{array}{rlrlrl}
\square_{c} u_{1} & =f & \text { in } \widetilde{\Omega}_{1} \times(0, T), & \square_{c} u_{2} & =f & \text { in } \widetilde{\Omega}_{2} \times(0, T), \\
u_{1} & =g & \text { on } \widetilde{\Gamma}_{10} \times(0, T), & u_{2} & =g & \text { on } \widetilde{\Gamma}_{20} \times(0, T), \\
u_{1}(\cdot, 0) & =u_{0} & \text { in } \widetilde{\Omega}_{1}, & u_{2}(\cdot, 0) & =u_{0} & \text { in } \widetilde{\Omega}_{2}, \\
\frac{\partial u_{1}}{\partial t}(\cdot, 0) & =u_{0}^{\prime} \text { in } \widetilde{\Omega}_{1}, & \frac{\partial u_{2}}{\partial t}(\cdot, 0) & =u_{0}^{\prime} \text { in } \widetilde{\Omega}_{2}, \\
u_{1} & =u_{2} \text { on } \widetilde{\Gamma}, & \frac{\partial u_{1}}{\partial n_{1}}+\frac{\partial u_{2}}{\partial n_{2}} & =0 & \text { on } \widetilde{\Gamma},
\end{array}
$$

where in the coupling conditions on the last line $n_{1}$ denotes the unit outward normal on $\widetilde{\Gamma}$ of $\Omega_{1}$ and $n_{2}$ denotes the unit outward normal on $\widetilde{\Gamma}$ of $\Omega_{2}$. The problems (2.1) and (3.1) are equivalent in the sense that $u_{1}$ is the restriction of $u$ on $\widetilde{\Omega}_{1}$ and $u_{2}$ is the restriction of $u$ to $\widetilde{\Omega}_{2}$.
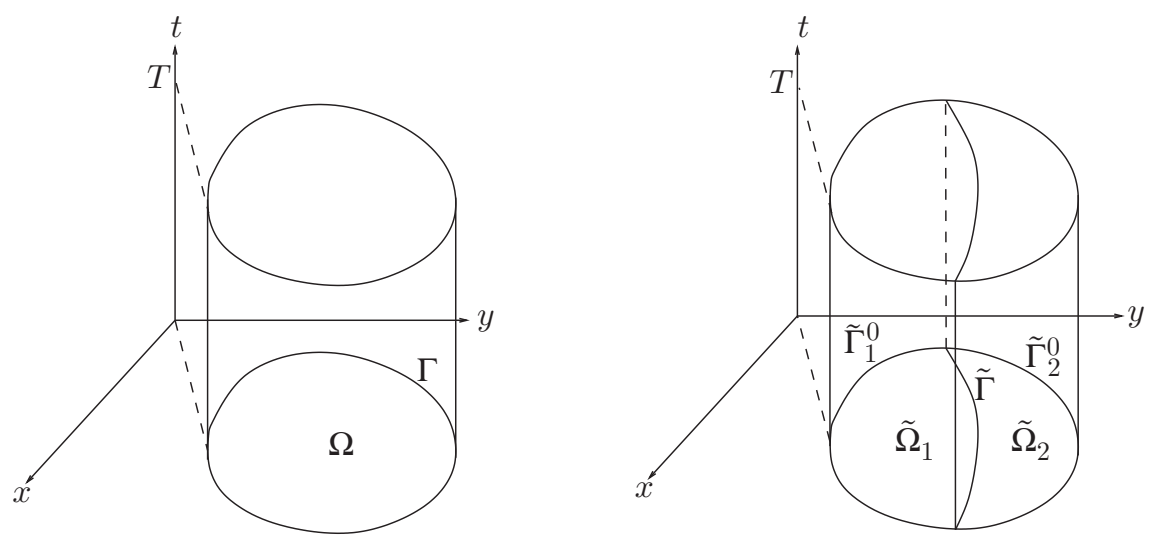

Figure 2. Entire domain $\Omega$ in space time on the left, and partitioned into two subdomains $\widetilde{\Omega}_{1}$ and $\widetilde{\Omega}_{2}$ on the right for a two dimensional example. 
We suppose now that the initial conditions $u_{0}$ and $u_{0}^{\prime}$, the boundary condition $g$ and the forcing function $f$ vanish in $\widetilde{\Omega}_{2}$. For a given $h$ in $L^{2}\left(0, T ; H_{00}^{\frac{1}{2}}(\widetilde{\Gamma})\right)$, we introduce for $w(\mathbf{x}, t)$ the problem

$$
\begin{array}{rll}
\square_{c} w & =0 & \text { in } \widetilde{\Omega}_{2} \times(0, T), \\
w & =0 & \text { on } \widetilde{\Gamma}_{20} \times(0, T), \\
w(\cdot, 0)=\frac{\partial w}{\partial t}(\cdot, 0) & =0 & \text { in } \widetilde{\Omega}_{2}, \\
w & =h & \text { on } \widetilde{\Gamma} .
\end{array}
$$

This problem has a unique solution $w \in L^{2}\left(0, T ; H^{1}\left(\widetilde{\Omega}_{2}\right)\right)$, and hence one can define the trace of the normal derivative of $w$ on $\widetilde{\Gamma}, \frac{\partial w}{\partial n_{2}}$. We define the operator $\mathcal{S}_{1, \widetilde{\Gamma}}^{e x}$ : $L^{2}\left(0, T ; H_{00}^{\frac{1}{2}}(\widetilde{\Gamma})\right) \mapsto L^{2}\left(0, T ;\left(H_{00}^{\frac{1}{2}}(\widetilde{\Gamma})\right)^{\prime}\right)$ by $\mathcal{S}_{1, \widetilde{\Gamma}}^{e x} h=\frac{\partial w}{\partial n_{2}}$, and since $\frac{\partial w}{\partial n_{2}}=-\frac{\partial w}{\partial n_{1}}, w$ solving (3.2) for any $h$ satisfies on $\widetilde{\Gamma} \times(0, T)$ the equation

$$
\frac{\partial w}{\partial n_{1}}+\mathcal{S}_{1, \widetilde{\Gamma}}^{e x} w=0
$$

This is the transparent condition for the subdomain $\widetilde{\Omega}_{1}$. The solution $u_{1}$ of the problem with the transparent condition

$$
\begin{aligned}
\square_{c} u_{1} & =f & & \text { in } \widetilde{\Omega}_{1} \times(0, T), \\
u_{1} & =g & & \text { on } \widetilde{\Gamma}_{10} \times(0, T), \\
u_{1}(\cdot, 0) & =u_{0}(\cdot) & & \text { in } \widetilde{\Omega}_{1}, \\
\frac{\partial u_{1}}{\partial t}(\cdot, 0) & =u_{0}^{\prime}(\cdot) & & \text { in } \widetilde{\Omega}_{1}, \\
\frac{\partial u_{1}}{\partial n_{1}}+\mathcal{S}_{1, \widetilde{\Gamma}}^{e x} u_{1} & =0 & & \text { on } \widetilde{\Gamma}
\end{aligned}
$$

coincides on $\Omega_{1}$ with $u$ solving (2.1). Similarly if the data vanish in $\Omega_{1}$, one defines

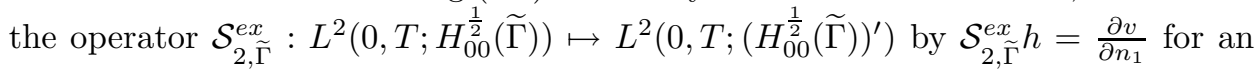
appropriate problem for $v$ and obtains the transparent condition

$$
\frac{\partial v}{\partial n_{2}}+\mathcal{S}_{2, \widetilde{\Gamma}}^{e x} v=0
$$

for the subdomain $\widetilde{\Omega}_{2}$. The operators

$$
\mathcal{B}_{1, \widetilde{\Gamma}}^{e x}:=\frac{\partial}{\partial n_{1}}+\mathcal{S}_{1, \widetilde{\Gamma}}^{e x}, \quad \mathcal{B}_{2, \widetilde{\Gamma}}^{e x}:=\frac{\partial}{\partial n_{2}}+\mathcal{S}_{2, \widetilde{\Gamma}}^{e x}
$$

are called transparent operators or exact transmission operators.

To gain more insight into the nature of the transparent operators, we identify the operators $\mathcal{S}_{2, \widetilde{\Gamma}}^{e x}$ and $\mathcal{S}_{1, \widetilde{\Gamma}}^{e x}$ in the case where the wave speed $c$ is constant and $\widetilde{\Omega}_{1}$ and $\widetilde{\Omega}_{2}$ are half spaces in $d$ spatial dimensions, $\widetilde{\Omega}_{1}=(-\infty, \delta) \times \mathbb{R}^{d-1}$ and $\widetilde{\Omega}_{2}=(\delta,+\infty) \times \mathbb{R}^{d-1}$ and the interface is $\widetilde{\Gamma}=\{\delta\} \times \mathbb{R}^{d-1}$. We use for the first spatial variable $x$ and for the remaining ones $\mathbf{y}=\left(y_{1}, \ldots, y_{d-1}\right) \in \mathbb{R}^{d-1}$. Our analysis is based on the Fourier transform in $\mathbf{y}$ and $t$,

$$
\hat{u}(x, k, \omega)=\frac{1}{(2 \pi)^{d / 2}} \int_{\mathbb{R}^{d}} u(x, \mathbf{y}, t) e^{-i(\omega t+\mathbf{k} \cdot \mathbf{y})} d y d t .
$$


Note that the integral in time is taken only for positive $t$ in the case of zero initial conditions. In the Fourier domain, problem (3.2) becomes

$$
\begin{aligned}
-\frac{\partial^{2} \hat{w}}{\partial x^{2}}+\left(|\mathbf{k}|^{2}-\frac{\omega^{2}}{c^{2}}\right) \hat{w} & =0 \quad \text { for } x \geq \delta, \\
\hat{w} & =\hat{h} \quad \text { at } x=\delta .
\end{aligned}
$$

We have to distinguish two cases: if $|\mathbf{k}|^{2}-\frac{\omega^{2}}{c^{2}} \geq 0$, then the unique solution of (3.7) which is not growing exponentially at infinity is an evanescent wave,

$$
\hat{w}=\hat{h} e^{-(x-\delta) \sqrt{|\mathbf{k}|^{2}-\frac{\omega^{2}}{c^{2}}}} .
$$

If however $|\mathbf{k}|^{2}-\frac{\omega}{c^{2}}<0$, then $\hat{w}$ is a priori composed of two propagating waves of the form $e^{ \pm i(x-\delta) \sqrt{\frac{\omega^{2}}{c^{2}}-|\mathbf{k}|^{2}}}$, which gives with the inverse Fourier transform a contribution to $w$ of the form $e^{+i\left(\omega t+\mathbf{k} \cdot \mathbf{y} \pm(x-\delta) \sqrt{\frac{\omega^{2}}{c^{2}}-|\mathbf{k}|^{2}}\right)}$. Since the initial conditions vanish, there can be no waves coming in from infinity in the $x$ direction, and hence the sign of $\omega\left( \pm \sqrt{\frac{\omega^{2}}{c^{2}}-|\mathbf{k}|^{2}}\right)$ must be positive. This determines the only propagating wave

$$
\hat{w}=\hat{h} e^{-i(x-\delta) \frac{\omega}{c} \sqrt{1-\frac{c^{2}|\mathbf{k}|^{2}}{\omega^{2}}}} .
$$

The symbol $\sigma_{1, \widetilde{\Gamma}}^{e x}$ of the operator $\mathcal{S}_{1, \widetilde{\Gamma}}^{e x}$ is in this special case independent of $\widetilde{\Gamma}$; it is given by

$$
\sigma_{1, \widetilde{\Gamma}}^{e x}=\sigma_{1}^{e x}= \begin{cases}\frac{|\omega|}{c} \sqrt{\frac{c^{2}|\mathbf{k}|^{2}}{\omega^{2}}-1} & \text { if }|\mathbf{k}|^{2}-\frac{\omega^{2}}{c^{2}} \geq 0, \\ i \frac{\omega}{c} \sqrt{1-\frac{c^{2}|\mathbf{k}|^{2}}{\omega^{2}}} & \text { if }|\mathbf{k}|^{2}-\frac{\omega^{2}}{c^{2}}<0,\end{cases}
$$

and we have $\hat{w}=\hat{h} e^{-\sigma_{1}^{e x}(x-\delta)}$. Similarly we find that the symbol $\sigma_{2, \widetilde{\Gamma}}^{e x}$ of the operator $\mathcal{S}_{2, \widetilde{\Gamma}}^{e x}$ does not depend on $\widetilde{\Gamma}$ and is given by

$$
\sigma_{2, \widetilde{\Gamma}}^{e x}=\sigma_{2}^{e x}= \begin{cases}\frac{|\omega|}{c} \sqrt{\frac{c^{2}|\mathbf{k}|^{2}}{\omega^{2}}-1} & \text { if }|\mathbf{k}|^{2}-\frac{\omega^{2}}{c^{2}} \geq 0, \\ i \frac{\omega}{c} \sqrt{1-\frac{c^{2}|\mathbf{k}|^{2}}{\omega^{2}}} & \text { if }|\mathbf{k}|^{2}-\frac{\omega^{2}}{c^{2}}<0,\end{cases}
$$

and we have $\hat{v}=\hat{h} e^{-\sigma_{2}^{e x}(x-\delta)}$. We see that in this special case the symbols given in (3.10) and (3.11) are the same and hence the two operators coincide: $\mathcal{S}_{1}^{e x}=\mathcal{S}_{2}^{e x}$. In general however the two operators are not the same and will depend on $\widetilde{\Gamma}$; see for example [20].

3.2. Approximate absorbing boundary conditions. The exact absorbing boundary conditions (3.3) and (3.5) contain the operators $\mathcal{S}_{1, \widetilde{\Gamma}}^{e x}$ and $\mathcal{S}_{2, \widetilde{\Gamma}}^{e x}$ which are, in general, nonlocal both in space and time because of the square root in their symbols. They are therefore costly in an implementation and approximations are of interest. To see what happens if an approximation is used, we consider the wave equation with constant wave speed $c$ on the half space $\widetilde{\Omega}_{1}, x<\delta$, with a linear boundary condition of the type

$$
\left(\partial_{n_{1}}+\mathcal{S}_{1, \widetilde{\Gamma}}\left(\partial_{t}, \partial_{y_{1}}, \partial_{y_{2}}, \ldots, \partial_{y_{d-1}}\right)\right) u=0 \quad \text { at } x=\delta .
$$


A propagating wave of the form $u^{I}=e^{i\left(\omega t+k_{x}(x-\delta)+\mathbf{k} \cdot \mathbf{y}\right)}$ with dispersion relation $\frac{\omega^{2}}{c^{2}}=k_{x}^{2}+|\mathbf{k}|^{2}$ propagates into the positive $x$ direction if $\omega k_{x}<0$, which implies

$$
\frac{c k_{x}}{\omega}=-\sqrt{1-\frac{c^{2}|\mathbf{k}|^{2}}{\omega^{2}}} .
$$

The wave $u^{I}$ is reflected by Descartes' law into a wave $R_{1} u_{1}^{R}$ where

$$
u_{1}^{R}=e^{i\left(\omega t-k_{x} x+\mathbf{k} \cdot \mathbf{y}\right)}
$$

and the reflection coefficient $R_{1}$ is defined by the equation

$$
\left(\partial_{n_{1}}+\mathcal{S}_{1, \widetilde{\Gamma}}\left(\partial_{t}, \partial_{y_{1}}, \partial_{y_{2}}, \ldots, \partial_{y_{d-1}}\right)\right)\left(u^{I}+R_{1} u_{1}^{R}\right)=0
$$

This leads to $R_{1}=\frac{i k_{x}+\sigma_{1, \tilde{\Gamma}}(i \omega, i \mathbf{k})}{i k_{x}-\sigma_{1, \tilde{\Gamma}}(i \omega, i \mathbf{k})}$, which becomes with 3.5

$$
R_{1}=\frac{\sigma_{2, \widetilde{\Gamma}}^{e x}(i \omega, i \mathbf{k})-\sigma_{1, \widetilde{\Gamma}}(i \omega, i \mathbf{k})}{\sigma_{2, \widetilde{\Gamma}}^{e x}(i \omega, i \mathbf{k})+\sigma_{1, \widetilde{\Gamma}}(i \omega, i \mathbf{k})}
$$

We see that if $\sigma_{1, \widetilde{\Gamma}}=\sigma_{2, \widetilde{\Gamma}}^{e x}$, then the reflection coefficient is identically zero and the boundary is completely transparent. The reflected wave at a distance $\delta$ from the boundary, in our case at $x=0$, is given by

$$
R_{1} e^{-\sigma_{2, \widetilde{\Gamma}}^{e x}(i \omega, i \mathbf{k}) \delta}
$$

which shows that for propagative waves, where $\sigma_{2, \widetilde{\Gamma}}^{e x}$ is imaginary, the amplitude is not reduced over the distance $\delta$, whereas for evanescent waves, $\sigma_{2, \widetilde{\Gamma}}^{e x}$ real positive, the amplitude decays exponentially in $\delta$. This important observation has an impact on the optimization in Section 5 .

Similarly in $\widetilde{\Omega}_{2}, x>\delta$, with a linear boundary condition

$$
\left(\partial_{n_{2}}+\mathcal{S}_{2, \widetilde{\Gamma}}\left(\partial_{t}, \partial_{y_{1}}, \partial_{y_{2}}, \ldots, \partial_{y_{d-1}}\right)\right) u=0 \quad \text { at } x=\delta,
$$

we find the reflection coefficient

$$
R_{2}=\frac{\sigma_{1, \widetilde{\Gamma}}^{e x}(i \omega, i \mathbf{k})-\sigma_{2, \widetilde{\Gamma}}(i \omega, i \mathbf{k})}{\sigma_{1, \widetilde{\Gamma}}^{e x}(i \omega, i \mathbf{k})+\sigma_{2, \widetilde{\Gamma}}(i \omega, i \mathbf{k})}
$$

and if $\sigma_{2, \widetilde{\Gamma}}=\sigma_{1, \widetilde{\Gamma}}^{e x}$, then the boundary is completely transparent. Including a layer $\delta$, we find for the reflected wave

$$
R_{2} e^{-\sigma_{1, \tilde{\Gamma}}^{e x}(i \omega, i \mathbf{k}) \delta}
$$

\section{Optimal SchWARZ WAVEForm RELAXATION}

We introduce now new transmission conditions in the overlapping Schwarz waveform relaxation algorithm (2.2). For two overlapping subdomains as given in Figure 3, we obtain

$$
\begin{aligned}
& \square_{c} u_{1}^{n}=f \quad \text { in } \Omega_{1} \times(0, T), \quad \square_{c} u_{2}^{n}=f \quad \text { in } \Omega_{2} \times(0, T), \\
& u_{1}^{n}=g \quad \text { on } \Gamma_{10} \times(0, T), \quad u_{2}^{n}=g \quad \text { on } \Gamma_{20} \times(0, T), \\
& u_{1}^{n}(\cdot, 0)=u_{0} \quad \text { in } \Omega_{1}, \quad u_{2}^{n}(\cdot, 0)=u_{0} \quad \text { in } \Omega_{2} \text {, } \\
& \frac{\partial u_{1}^{n}}{\partial t}(\cdot, 0)=u_{0}^{\prime} \quad \text { in } \Omega_{1}, \quad \frac{\partial u_{2}^{n}}{\partial t}(\cdot, 0)=u_{0}^{\prime} \quad \text { in } \Omega_{2} \text {, } \\
& \mathcal{B}_{1, \Gamma_{1}} u_{1}^{n}=\mathcal{B}_{1, \Gamma_{1}} u_{2}^{n-1} \text { on } \Gamma_{1}, \quad \mathcal{B}_{2, \Gamma_{2}} u_{2}^{n}=\mathcal{B}_{2, \Gamma_{2}} u_{1}^{n-1} \text { on } \Gamma_{2},
\end{aligned}
$$




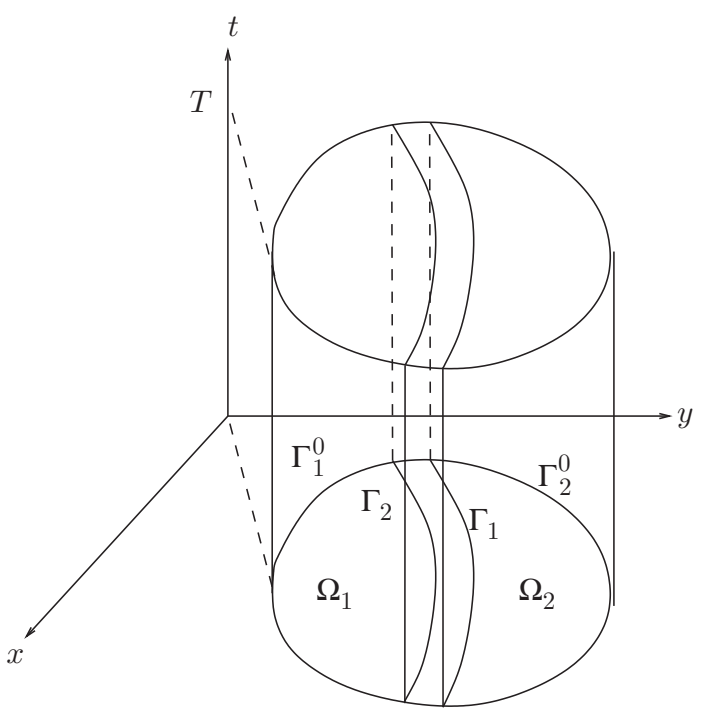

FiguRE 3. Decomposition into two subdomains.

where $\mathcal{B}_{1, \Gamma_{1}}$ and $\mathcal{B}_{2, \Gamma_{2}}$ are general operators acting in the tangential and time dimension at the interfaces and leading to well-posed subdomain problems. We investigate in the sequel which operators lead to fast algorithms. By linearity it suffices in the analysis of (4.1) to consider the homogeneous problems, $f=g=u_{0}=u_{0}^{\prime}=0$, and to analyze convergence to zero. This is because the error equations coincide with these homogeneous equations. Using (3.3), $u_{2}^{n}$ satisfies for any $n \geq 1$ on $\Gamma_{2}$ the equation

$$
\mathcal{B}_{1, \Gamma_{1}}^{e x} u_{2}^{n}=\frac{\partial u_{2}^{n}}{\partial n_{1}}+\mathcal{S}_{1, \Gamma_{1}}^{e x} u_{2}^{n}=0
$$

where $\mathcal{S}_{1, \Gamma_{1}}^{e x}$ is the transparent operator for $\Omega_{1}$. Similarly for any $n \geq 1, u_{1}^{n}$ satisfies according to (3.5) the equation

$$
\mathcal{B}_{2, \Gamma_{2}}^{e x} u_{1}^{n}=\frac{\partial u_{1}^{n}}{\partial n_{2}}+\mathcal{S}_{2, \Gamma_{2}}^{e x} u_{1}^{n}=0
$$

where $\mathcal{S}_{2, \Gamma_{2}}^{e x}$ is the transparent operator for $\Omega_{2}$.

Theorem 4.1. If $\mathcal{B}_{1, \Gamma_{1}}:=\mathcal{B}_{1, \Gamma_{1}}^{e x}$ and $\mathcal{B}_{2, \Gamma_{2}}:=\mathcal{B}_{2, \Gamma_{2}}^{\text {ex }}$, then $u_{1}^{2} \equiv u$ in $\Omega_{1} \times(0, T)$ and $u_{2}^{2} \equiv u$ in $\Omega_{2} \times(0, T)$ for any $T$ and hence the algorithm converges in exactly two steps with the transparent transmission conditions.

Proof. The proof is the same as in one dimension; see [20].

This result generalizes in two ways. First, the algorithm converges globally in time in $J$ steps for $J$ subdomains, provided that the subdomains form a chain in space and the exact absorbing transmission conditions are used; see for example 20]. Second, this result holds for many types of equations; it was shown for example for a steady convection diffusion problem in [39].

Remark 4.2. On bounded time intervals, the optimized Schwarz waveform relaxation algorithm (4.1) for the wave equation converges at least as well as the classical Schwarz waveform relaxation algorithm (2.2), since the proof of Theorem [2.1] still 
applies. On short time intervals, it will in general converge in less than $J$ steps for $J$ subdomains because of this. For an analysis in the one dimensional case, see [20].

To learn more about the behavior of the algorithm (4.1) for general transmission conditions $\mathcal{B}_{i, \widetilde{\Gamma}}, i=1,2$, we consider now the case of constant wave speed $c$ and two half spaces with overlap, $\Omega_{1}=(-\infty, \delta) \times \mathbb{R}^{d-1}$ and $\Omega_{2}=(0,+\infty) \times \mathbb{R}^{d-1}$. We use transmission conditions of the form

$$
\begin{aligned}
& \mathcal{B}_{1, \Gamma_{1}}=\partial_{n_{1}}+\mathcal{S}_{1, \Gamma_{1}}\left(\partial_{t}, \partial_{y_{1}}, \partial_{y_{2}}, \ldots, \partial_{y_{d-1}}\right), \\
& \mathcal{B}_{2, \Gamma_{2}}=\partial_{n_{2}}+\mathcal{S}_{2, \Gamma_{2}}\left(\partial_{t}, \partial_{t}, \partial_{y_{1}}, \partial_{y_{2}}, \ldots, \partial_{y_{d-1}}\right) .
\end{aligned}
$$

Taking a Fourier transform in $\mathbf{y}$ and $t$ of the algorithm (4.1) with homogeneous data (the error equations), we obtain

$$
\begin{aligned}
-\frac{\partial^{2} \hat{u}_{1}^{n}}{\partial x^{2}}+\left(|\mathbf{k}|^{2}-\frac{\omega^{2}}{c^{2}}\right) \hat{u}_{1}^{n} & =0 & & \text { for } x<\delta, \\
\left(\frac{\partial}{\partial x}+\sigma_{1, \Gamma_{1}}(i \omega, i \mathbf{k})\right) \hat{u}_{1}^{n} & =\left(\frac{\partial}{\partial x}+\sigma_{1, \Gamma_{1}}(i \omega, i \mathbf{k})\right) \hat{u}_{2}^{n-1} & & \text { on } x=\delta, \\
-\frac{\partial^{2} \hat{u}_{2}^{n}}{\partial x^{2}}+\left(|\mathbf{k}|^{2}-\frac{\omega^{2}}{c^{2}}\right) \hat{u}_{1}^{n} & =0 & & \text { for } x>0, \\
\left(-\frac{\partial}{\partial x}+\sigma_{2, \Gamma_{2}}(i \omega, i \mathbf{k})\right) \hat{u}_{2}^{n} & =\left(-\frac{\partial}{\partial x}+\sigma_{2, \Gamma_{2}}(i \omega, i \mathbf{k})\right) \hat{u}_{1}^{n-1} & & \text { on } x=0 .
\end{aligned}
$$

Using equations (3.10) and (3.11), we obtain the iterates

$$
\hat{u}_{1}^{n}=\hat{a}^{n} e^{\sigma_{2, \Gamma_{2}}^{e x}(x-\delta)}, \quad \hat{u}_{2}^{n}=\hat{b}^{n} e^{-x \sigma_{1, \Gamma_{1}}^{e x}}
$$

and with the transmission conditions from (4.3) to determine the constants $\hat{a}^{n}$ and $\hat{b}^{n}$, we find

$$
\begin{aligned}
& \hat{a}^{n}=\frac{-\sigma_{2, \Gamma_{2}}^{e x}(i \omega, i \mathbf{k})+\sigma_{1, \Gamma_{1}}(i \omega, i \mathbf{k})}{\sigma_{1, \Gamma_{1}}^{e x}(i \omega, i \mathbf{k})+\sigma_{1, \Gamma_{1}}(i \omega, i \mathbf{k})} e^{-\delta \sigma_{2, \Gamma_{2}}^{e x}(i \omega, i \mathbf{k})} \hat{b}^{n-1}, \\
& \hat{b}^{n}=\frac{-\sigma_{1, \Gamma_{1}}^{e x}(i \omega, i \mathbf{k})+\sigma_{2, \Gamma_{2}}(i \omega, i \mathbf{k})}{\sigma_{2, \Gamma_{2}}^{e x}(i \omega, i \mathbf{k})+\sigma_{2, \Gamma_{2}}(i \omega, i \mathbf{k})} e^{-\delta \sigma_{1, \Gamma_{1}}^{e x}(i \omega, i \mathbf{k})} \hat{a}^{n-1} .
\end{aligned}
$$

Using one equation at step $n-1$ in the other, we find $\hat{a}^{n}=\rho \hat{a}^{n-2}$ and $\hat{b}^{n}=\rho \hat{b}^{n-2}$, where the convergence rate $\rho$ is given by

$$
\rho:=\frac{\sigma_{2, \Gamma_{2}}^{e x}-\sigma_{1, \Gamma_{1}}}{\sigma_{1, \Gamma_{1}}^{e x}+\sigma_{1, \Gamma_{1}}} \cdot \frac{\sigma_{1, \Gamma_{1}}^{e x}-\sigma_{2, \Gamma_{2}}}{\sigma_{2, \Gamma_{2}}^{e x}+\sigma_{2, \Gamma_{2}}} e^{-\delta\left(\sigma_{1, \Gamma_{1}}^{e x}+\sigma_{2, \Gamma_{2}}^{e x}\right)} .
$$

The quantity $\rho$ is sometimes also called the convergence factor, but we use in the sequel the term convergence rate for $\rho$. The factors in the convergence rate (4.6) have a strong resemblance to the reflection coefficients $R_{1}$ defined in (3.14), (3.15) and $R_{2}$ defined in (3.17), (3.18), including the exponential coming in for the reflected wave at a distance $\delta$ from the boundary. There is however a subtle difference: in the denominator the symbols of the exact operators are exchanged. Hence for approximations of the absorbing boundary conditions, the optimization will depend on their purpose: for domain decomposition, one will try to minimize the convergence rate $\rho$; to truncate domains, one will try to minimize the reflection coefficients $R_{j}$ which leads in general to a different, although related, optimization problem. In the case of half spaces however, we have seen that $\sigma_{1, \Gamma_{1}}^{e x}=\sigma_{2, \Gamma_{2}}^{e x}$ and therefore the two optimization problems coincide in that case. In the sequel we further analyze this special case, and thus denote it by $\sigma^{e x}:=\sigma_{1, \Gamma_{1}}^{e x}=\sigma_{2, \Gamma_{2}}^{e x}$. 


\section{Optimized ABSORBING CONDITIONS}

For propagative waves, where the symbol $\sigma^{e x}$ is imaginary, the overlap is not effective in the convergence rate $\rho$ given in (4.6), since the modulus of the exponential is one. This does not contradict Theorem 2.1 because our analysis here is for all time, whereas the result of Theorem 2.1 is valid for bounded time intervals only. To treat propagative waves effectively, we need to choose operators in the transmission conditions with symbol $\sigma_{j}, j=1,2$, that are close to $\sigma^{e x}$. We will therefore choose in the sequel $\sigma_{j}$ to be imaginary, although other choices are possible. If $\sigma_{j}$ is purely imaginary, then for evanescent waves the modulus of the fraction in front of the exponential in the convergence rate (4.6) equals one. But for evanescent waves the modulus of the exponential is less than one. Hence for the choice of $\sigma_{j}$ imaginary, evanescent waves are treated effectively by the overlap, whereas propagative waves can be treated by the transmission conditions. The same observation holds for the reflected waves at the distance $\delta$ from the absorbing boundary, because of the relation between the convergence rate (4.6) and the reflected waves (3.15) and (3.18).

5.1. Approximation by a rational function. To approximate the transparent operator $\mathcal{B}_{1}^{\text {ex }}$ (the operator $\mathcal{B}_{2}^{\text {ex }}$ is similar), we approximate the symbol

$$
\sigma^{e x}=i \frac{\omega}{c} \sqrt{1-\frac{c^{2}|\mathbf{k}|^{2}}{\omega^{2}}} \approx i \frac{\omega}{c} r\left(\frac{c|\mathbf{k}|}{\omega}\right)=: \sigma_{j}^{a p p}, \quad j=1,2
$$

where $r$ is a rational function. Padé approximations have been proposed first in [15] and several generalizations followed. We use in the sequel the notation established in 44].

We assume that the rational fraction $r(s)=\frac{p_{m}(s)}{q_{\widetilde{m}}(s)}$ is such that the polynomial $p_{m}$ is of degree $m$, the polynomial $q_{\widetilde{m}}$ is of degree $\widetilde{m}$ and they have no common factors. The symbol of the associated operator is

$$
\beta_{1}^{a p p}=\frac{\partial}{\partial x}+i \frac{\omega}{c} r\left(\frac{c|\mathbf{k}|}{\omega}\right) .
$$

The associated approximately absorbing boundary conditions, which are differential operators, are obtained by multiplying through by $\omega^{\bar{m}-1} q_{\widetilde{m}}\left(\frac{c|\mathbf{k}|}{\omega}\right)$, where $\bar{m}=\max \{m, \widetilde{m}+1\}$. We thus obtain

$$
\begin{aligned}
& \tilde{\beta}_{1}^{a p p}=\omega^{\bar{m}-1} q_{\widetilde{m}}\left(\frac{c|\mathbf{k}|}{\omega}\right) \frac{\partial}{\partial x}+i \frac{\omega}{c} p_{m}\left(\frac{c|\mathbf{k}|}{\omega}\right), \\
& \tilde{\beta}_{2}^{a p p}=-\omega^{\bar{m}-1} q_{\widetilde{m}}\left(\frac{c|\mathbf{k}|}{\omega}\right) \frac{\partial}{\partial x}+i \frac{\omega}{c} p_{m}\left(\frac{c|\mathbf{k}|}{\omega}\right) .
\end{aligned}
$$

The symbols $\tilde{\beta}_{j}^{a p p}, j=1,2$, are the symbols of partial differential operators $\widetilde{\mathcal{B}}_{j}^{a p p}$ in $d+1$ variables of global degree $\bar{m}$ and first degree in $x$ with real coefficients. We associate with the operator $\widetilde{\mathcal{B}}_{1}^{\text {app }}$ a boundary value problem in the half space $\Omega_{1}=(-\infty, \delta) \times \mathbb{R}^{d-1}$,

$$
\begin{array}{rlll}
\square_{c} u_{1} & =f & \text { in } \Omega_{1} \times(0, T), \\
u_{1}(\cdot, 0) & =u_{0} & \text { in } \Omega_{1}, \\
\frac{\partial u_{1}}{\partial t}(\cdot, 0) & =u_{0}^{\prime} & \text { in } \Omega_{1}, \\
\widetilde{\mathcal{B}}_{1}^{a p p}\left(\partial_{t}, \partial_{x}, \partial_{y_{1}}, \partial_{y_{2}}, \ldots, \partial_{y_{d-1}}\right) u_{1} & =h \quad & \text { on }\{\delta\} \times \mathbb{R}^{d-1} \times(0, T),
\end{array}
$$


and similarly for $\widetilde{\mathcal{B}}_{2}^{a p p}$. We say that the problem is well posed in the sense of Kreiss if there exists a unique solution whose norm in time $t$ inside the domain and on the boundary can be estimated by the data. The following result can be found in [44].

Theorem 5.1. The boundary value problem (5.2) is well posed in the sense of Kreiss if and only if the zeros and the poles of $r(s) / s$ are real, simple, interlace on the real axis and if in addition $r(s)>0$ for $s$ in the interval $[-1,1]$.

The reflection coefficient is then given by

$$
R_{1}(i \omega, i \mathbf{k})=R_{2}(i \omega, i \mathbf{k})=\frac{\sqrt{1-s^{2}}-r(s)}{\sqrt{1-s^{2}}+r(s)}, \quad s=\frac{c|\mathbf{k}|}{\omega} .
$$

We assume in the sequel that the rational function $r(s)$ satisfies the hypotheses of Theorem 5.1. We can therefore associate with the rational function $r$ the domain decomposition algorithm (4.1) on the subdomains $\Omega_{1}=(-\infty, \delta] \times \mathbb{R}^{d-1}$ and $\Omega_{2}=$ $(0,+\infty) \times \mathbb{R}^{d-1}$, with the transmission conditions

$$
\mathcal{B}_{j}:=\widetilde{\mathcal{B}}_{j}^{a p p}, \quad j=1,2 .
$$

Corollary 5.2. If the boundary value problem (5.2) is well posed, then $\widetilde{m} \leq m \leq$ $\widetilde{m}+2$. If $\widetilde{m} \leq m \leq \widetilde{m}+2$ and $r(s)$ interpolates $\sqrt{1-s^{2}}$ at $m+\widetilde{m}+1+\chi_{m \widetilde{m}}$ points counted with their multiplicities, where

$$
\chi_{m \widetilde{m}}= \begin{cases}0 & \text { if } m=\widetilde{m} \text { is odd, } \\ 1 & \text { if } m=\widetilde{m} \text { is even, }\end{cases}
$$

then the boundary value problem (5.2) is well posed.

Proof. This is a consequence of Theorem [5.1, a detailed proof can be found in 44].

It is natural to choose $m$ and $\widetilde{m}$ even, since the function to be approximated is even. Following [40], we let $2 L \geq 2$ be the number of points $s_{i}$ in $[-1,1]$, $\pm s_{1}, \ldots, \pm s_{L}$, counted with their multiplicities. The points \pm 1 can be included, but then the boundary value problem is weakly ill-posed, since then there are tangential waves along the boundary. Setting $m=2\left\lfloor\frac{L}{2}\right\rfloor$ and $\widetilde{m}=2\left\lceil\frac{L}{2}\right\rceil-2$, we obtain $2 L=m+\widetilde{m}+2$ and letting $p(\tau)=\prod_{l=1}^{L}\left(\tau-\sqrt{1-s_{l}^{2}}\right)$, we define the rational function $r$ on $[0,1]$ by

$$
r(s):=\tau \frac{p(-\tau)+p(\tau)}{p(-\tau)-p(\tau)}, \quad \text { where } \tau=\sqrt{1-s^{2}} .
$$

A simple calculation shows that $r$ interpolates $\sqrt{1-s^{2}}$ at the points $\pm s_{l}$. The reflection coefficients (5.3) become $R_{1}(i \omega, i \mathbf{k})=R_{2}(i \omega, i \mathbf{k})=R\left(\sqrt{1-s^{2}}\right)$ where $R$ defined on $[-1,1]$ is given by

$$
R(\tau)=\frac{p(\tau)}{p(-\tau)}=\prod_{l=1}^{L} \frac{\tau-\tau_{l}}{\tau+\tau_{l}}, \quad \tau_{l}=\sqrt{1-s_{l}^{2}}
$$

and we therefore have $\left|R_{1}\right|=\left|R_{2}\right| \leq 1$. For the modulus of the convergence rate $\rho$ given in (4.6) we find

$$
|\rho|= \begin{cases}\left|\rho_{p}\right|=\prod_{l=1}^{L}\left|\frac{\tau-\tau_{l}}{\tau+\tau_{l}}\right|^{2}, \tau=\sqrt{1-\left(\frac{c|\mathbf{k}|}{\omega}\right)^{2}}, & \text { for propagative waves, }|c \mathbf{k} / \omega| \leq 1, \\ \left|\rho_{e}\right|=e^{-2 \delta \sqrt{|\mathbf{k}|^{2}-\omega^{2} / c^{2}}}, & \text { for evanescent waves, }|c \mathbf{k} / \omega| \geq 1\end{cases}
$$

and thus the convergence rate satisfies $|\rho| \leq 1$ for all $k, \omega$. 
Theorem 5.3. The subdomain problems in algorithm (4.1) with transmission conditions defined by the symbols (5.1) where the rational functions are given by (5.6) are well posed in the sense of Kreiss and the algorithm converges. For any initial guess in $L^{2}\left(0, T ; H^{1}\left(\Omega_{1}\right)\right) \times L^{2}\left(0, T ; H^{1}\left(\Omega_{2}\right)\right)$ we have

$$
\lim _{n \rightarrow \infty}\left\|u_{i}^{n}\right\|_{L^{2}\left((0, T) \times \Omega_{i}\right)}=0, \quad i=1,2 .
$$

Proof. The well posedness follows from Corollary 5.2] and is shown in [44. To prove convergence of the algorithm (4.1), we show the argument without loss of generality for the first subdomain and for even iterates only. Extending $u_{1}^{2 p}$ by 0 on $(T, \infty)$ and integrating (4.4), we obtain

$$
\left\|u_{1}^{2 p}\right\|_{L^{2}\left((0, T) \times \Omega_{1}\right)}^{2}=\int_{-\infty}^{\delta} \int_{\mathbb{R}^{d}}|\rho|^{2 p}\left|u_{1}^{0}\right|^{2} d x d \omega d \mathbf{k} .
$$

Following [15, we distinguish three regions in the plane $(\omega,|\mathbf{k}|)$. For a given $\mu>0$, we define the glancing region $S_{g}:=\left\{(\mathbf{k}, \omega): 1-\mu \leq \frac{c^{2}|\mathbf{k}|^{2}}{\omega^{2}} \leq 1+\mu\right.$ or $c^{2}|\mathbf{k}|^{2}+\omega^{2}<$ $\mu\}$, the propagating region $S_{p}:=\left\{(\mathbf{k}, \omega): \frac{c^{2}|\mathbf{k}|^{2}}{\omega^{2}} \leq 1-\mu\right.$ and $\left.c^{2}|\mathbf{k}|^{2}+\omega^{2}>\mu\right\}$ and the evanescent region $S_{e}:=\left\{(\mathbf{k}, \omega): \frac{c^{2}|\mathbf{k}|^{2}}{\omega^{2}} \geq 1+\mu\right.$ and $\left.c^{2}|\mathbf{k}|^{2}+\omega^{2}>\mu\right\}$, as shown in Figure 4 on the left. We first look at the integral in the glancing region,

$$
\int_{-\infty}^{\delta} \int_{S_{g}}\left|u_{1}^{0}\right|^{2} d x d \omega d \mathbf{k}
$$

Since the initial guess $u_{1}^{0}$ is in $L^{2}$, we can choose for any $\epsilon>0$ the parameter $\mu$ such that the integral is less than $\epsilon$. If in addition $\mu$ is small enough such that in the reflecting region all $\tau_{l}$ are bigger than $\mu^{1 / 4}$, then we find from (5.8) after a short calculation

$$
\sup _{S_{p}}|\rho(\omega, \mathbf{k})| \leq \prod_{l=1}^{L}\left|\frac{\sqrt{\mu}-\tau_{l}}{\sqrt{\mu}+\tau_{l}}\right|^{2}=\left|\rho_{p}\right|<1 .
$$

In the evanescent region, we obtain from the two inequalities $\frac{c^{2}|\mathbf{k}|^{2}}{\omega^{2}}>1+\mu$ and $c^{2}|\mathbf{k}|^{2}+\omega^{2}>\mu$ the lower bound $c^{2}|\mathbf{k}|^{2}-\omega^{2}>\frac{2-\mu}{2+\mu}$ (see Figure $\llbracket$ on the right) and
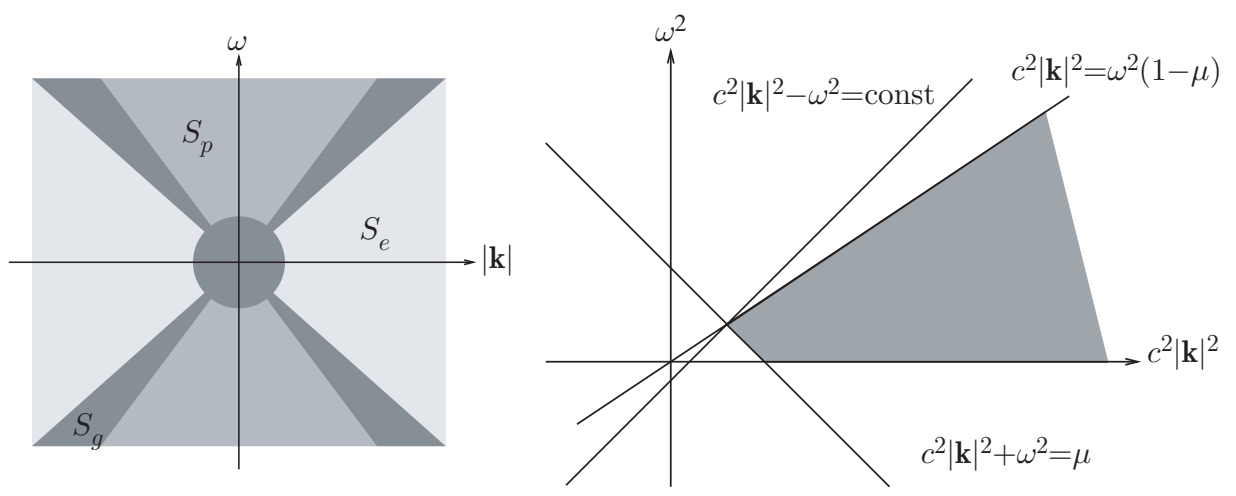

Figure 4 . On the left the glancing, propagating and evanescent regions in the $(\omega,|\mathbf{k}|)$ plane, and on the right a drawing for the proof of Theorem 5.3 
hence with (5.8) the bound on the convergence rate

$$
\sup _{S_{e}}|\rho(\omega, \mathbf{k})| \leq e^{-\frac{2 \delta}{c} \sqrt{\frac{2-\mu}{2+\mu}}}=\left|\rho_{e}\right|<1 .
$$

Therefore for $\mu$ sufficiently small we obtain by the causality principle

$\left\|u_{1}^{2 p}\right\|_{L^{2}\left((0, T) \times \Omega_{1}\right)}^{2} \leq \epsilon+\left|\rho_{p}\right|^{2 p} \int_{-\infty}^{\delta} \int_{S_{p}}\left\|u_{1}^{0}\right\|^{2} d x d \omega d \mathbf{k}+\left|\rho_{e}\right|^{2 p} \int_{-\infty}^{\delta} \int_{S_{e}}\left\|u_{1}^{0}\right\|^{2} d x d \omega d \mathbf{k}$ and thus the method converges.

5.2. Minimizing the reflection coefficient. Classical optimizations of the absorbing boundary conditions are based on the study of the error between the rational function $r(s)$ and the symbol $\sqrt{1-s^{2}}$; see for example [44. We propose here a different strategy which is based on the minimization of the reflection coefficient and includes an overlap, motivated by the Schwarz waveform relaxation algorithm. If we introduce a layer of width $\delta$ at the boundary, then evanescent waves are effectively damped. Furthermore, for propagating waves to be reflected and to enter the domain of interest again, they have to cross this layer twice, first to get to the boundary and then back to get into the domain. A propagating wave arriving under the angle $\theta$ at the interface with wave speed $c$ will therefore need the time $t=\frac{2 \delta}{c \cos \theta}$ to get through the layer to the absorbing boundary and back; see Figure 5 Hence on a simulation interval $[0, T]$ a wave does not need to be absorbed by the absorbing boundary condition, if the angle of incidence satisfies

$$
\theta \geq \theta_{\max }=\arccos \frac{2 \delta}{c T}
$$

because such a wave cannot get back into the domain of interest. We therefore optimize the absorbing boundary conditions in the $L^{\infty}$ norm on the subinterval $[\underline{\tau}, 1] \subset[0,1]$ where $\underline{\tau}:=\cos \theta_{\max }$. This leads to the min-max problem for the reflection coefficient $R(\tau)$,

$$
\min _{\underline{\tau} \leq \tau_{1}, \ldots, \tau_{L} \leq 1}\left(\sup _{\tau \in[\tau, 1]}|R(\tau)|\right)=\min _{\underline{\tau} \leq \tau_{1}, \ldots, \tau_{L} \leq 1}\left(\sup _{\tau \in[\tau, 1]} \prod_{l=1}^{L}\left|\frac{\tau-\tau_{l}}{\tau+\tau_{l}}\right|\right) .
$$

Lemma 5.4. The solution of the min-max problem (5.10) for $L=1$ is $\tau_{1}=\sqrt{\underline{\tau}}$. For $L=2$ the solution is

$$
\tau_{1}=\frac{\sqrt{2} \underline{\tau}^{3 / 4}}{\sqrt{1+\underline{\tau}}+\sqrt{\underline{\tau}}-1}, \quad \tau_{2}=\frac{\underline{\tau}^{1 / 4}(\sqrt{1+\underline{\tau}}+\sqrt{\underline{\tau}}-1)}{\sqrt{2}} .
$$

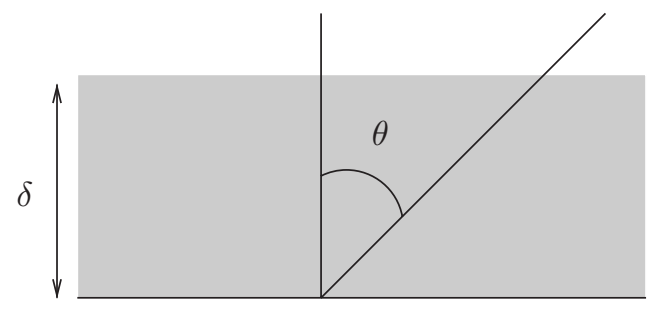

FiguRE 5. Angle of incidence of a propagating wave onto the interface, traversing the layer of width $\delta$. 
Proof. For $L=1$ we first note that $R(\tau)$ is monotone in $\tau$ and hence the maximum must be attained on the boundary in the min-max problem. A derivative with respect to $\tau_{1}$ shows that the optimum is reached when we balance at the boundary, $R\left(\underline{\tau}, \tau_{1}\right)=-R\left(0, \tau_{1}\right)$. This equation has the solution $\tau_{1}=\sqrt{\underline{\tau}}$.

For $L=2$ the optimization problem for a given $\underline{\tau}$ is the min-max problem

$$
\min _{\underline{\tau} \leq \tau_{1} \leq \tau_{2} \leq 1}\left(\sup _{\underline{\tau} \leq \tau \leq 1}\left|\frac{\tau-\tau_{1}}{\tau+\tau_{1}}\right|\left|\frac{\tau-\tau_{2}}{\tau+\tau_{2}}\right|\right) .
$$

Because of the absolute value, the function is always nonnegative. It is zero for $\tau=\tau_{1}$ and $\tau=\tau_{2}$. Computing the derivative with respect to $\tau$, we find that the function has a maximum between $\tau_{1}$ and $\tau_{2}$ at the geometric mean $\tau=\sqrt{\tau_{1} \tau_{2}}$. In addition for $\tau<\tau_{1}$ the function is decreasing in $\tau_{1}$, whereas for $\tau_{1}<\tau<\tau_{2}$ it increases when $\tau_{1}$ decreases. Similarly for $\tau>\tau_{2}$ the function is increasing if $\tau_{2}$ decreases and for $\tau_{1}<\tau<\tau_{2}$ it is decreasing when $\tau_{2}$ decreases. Therefore the solution of the min-max problem is obtained when the values of the function at $\tau=\underline{\tau}, \tau=\sqrt{\tau_{1} \tau_{2}}$ and $\tau=1$ are equal. This leads to the system of equations

$$
\frac{\left(\tau_{1}-\underline{\tau}\right)\left(\tau_{2}-\underline{\tau}\right)}{\left(\tau_{1}+\underline{\tau}\right)\left(\tau_{2}+\underline{\tau}\right)}=\frac{\left(\sqrt{\tau_{2}}-\sqrt{\tau_{1}}\right)^{2}}{\left(\sqrt{\tau_{2}}+\sqrt{\tau_{1}}\right)^{2}}, \quad \frac{\left(\tau_{1}-\underline{\tau}\right)\left(\tau_{2}-\underline{\tau}\right)}{\left(\tau_{1}+\underline{\tau}\right)\left(\tau_{2}+\underline{\tau}\right)}=\frac{\left(1-\tau_{1}\right)\left(1-\tau_{2}\right)}{\left(1+\tau_{1}\right)\left(1+\tau_{2}\right)},
$$

which has the solution given in the lemma.

While the preceding lemma gives the optimal parameters to use in an absorbing boundary condition of first or second order with a layer of width $\delta$, there is no closed form solution in general for the optimal parameters $\tau_{j}$. There is however a general existence and uniqueness result and the optimum is characterized by the equioscillation principle. In the special case where the order $L$ is a power of 2 , there is a recursive formula for the optimal parameters; see [45].

5.3. Minimizing the convergence rate. As in the case of the reflection coefficient, evanescent waves are damped by the overlap $\delta$ of the optimized Schwarz waveform relaxation algorithm. We can therefore focus on minimizing the convergence rate $\rho$ given in (4.6) for propagating waves only, and for those we have for our rational approximation $\sqrt{|\rho|}=|R(\tau)|$ where $R(\tau)$ is the reflection coefficient defined in (5.7) (the square root comes from the fact that we defined the convergence rate over a double step of the algorithm). In contrast to the optimization of the reflection coefficient however, $\theta_{\max }$ is not fixed because of the iteration. A wave arriving under the angle $\theta$ on the interface of a subdomain has taken the time $t=\frac{\delta}{c \cos \theta}$ to travel across the overlap of size $\delta$. Hence this wave is of no importance in the first iteration for a simulation on a time interval $[0, T]$ if

$$
\theta \geq \theta_{\max }^{1}=\arccos \frac{\delta}{c T} .
$$

Thus waves arriving close to the tangent to the interface will be treated in the first iteration already by the overlap. In the second iteration, a wave needs to travel twice across the overlap to have an influence, and hence it is of no importance in a simulation on $[0, T]$ if

$$
\theta \geq \theta_{\max }^{2}=\arccos \frac{2 \delta}{c T}
$$

and $\theta_{\max }^{2}<\theta_{\max }^{1}$. Hence after two steps more waves have been treated effectively by the overlap than after one step only. In general, at iteration step $n$, a wave 
arriving at the angle $\theta$ on the interface can be neglected in the optimization if

$$
\theta \geq \theta_{\max }^{n}=\arccos \frac{n \delta}{c T} .
$$

We see again that for $n>c T / \delta, \theta_{\max }^{n}$ has reached 0 and all the waves have converged, as in the case of Dirichlet transmission conditions in Theorem 2.1. The overlap is therefore not only good for evanescent waves, it is also effective for waves with large $\theta$ and the transmission conditions only need to be effective for the waves with small $\theta$, the waves that cross the overlap rapidly. This is ideal, because for those waves the transmission conditions are effective, whereas for waves parallel to the interface, the transmission conditions are not effective.

A first simple and effective choice for the optimization parameters is thus to absorb all waves coming in orthogonally to the interface, $\tau_{1}=\tau_{2}=\ldots=\tau_{L}=1$. With such an approximate absorbing transmission condition, orthogonal waves are completely absorbed, and the more a wave arrives at an oblique angle, the less it is absorbed, but the earlier it is removed by the overlap. This approach is very convenient, since the optimization parameters are known for all $L$ and no further information is needed to run the algorithm.

If we know however a priori a given tolerance $\epsilon$ for the precision we want to obtain, then we can do a different optimization.

Theorem 5.5 (Minimum number of iterations). To reach a precision $\epsilon$ for the propagating waves, the minimum number of iterations the optimized Schwarz waveform relaxation algorithm with transmission conditions of order $L$ needs is $\lceil n\rceil$ where $n$ solves

$$
\left(\rho_{K}\left(\theta_{\max }^{n}\right)\right)^{n}=\epsilon, \quad \theta_{\max }^{n}=\arccos \frac{n \delta}{c T}
$$

with $\rho_{K}\left(\theta_{\max }^{n}\right)$ denoting the maximum in the solution of the min-max problem (5.10) .

Proof. To achieve the given precision $\epsilon$ in $n$ iterations, the angle $\theta_{\max }^{n}$ needs to be such that all waves arriving under an angle bigger than $\theta_{\max }^{n}$ have been eliminated: $\theta_{\max }^{n} \geq \arccos \frac{n \delta}{c T}$. In addition waves arriving at an angle smaller than $\theta_{\max }^{n}$ have been reduced by the optimized convergence rate below the tolerance $\epsilon$ : $\left(\rho_{K}\left(\theta_{\max }^{n}\right)\right)^{n} \leq \epsilon$. The result of the theorem follows from these two observations.

Once $n$ has been determined, we know the angle $\theta_{\max }^{n}$ and hence the optimal parameters to be used in the transmission conditions which minimize the convergence rate uniformly in the interval $[\underline{\tau}, 1]$ from Lemma [5.4

In practical calculations convergence below the discretization error is not of interest; hence $\epsilon=C_{1} h^{p}$ where $p$ denotes the order of the numerical scheme and $C_{1}$ is some positive constant. In addition one can rarely afford an overlap which is independent of $h$; usually it is a few grid cells, $\delta=C_{2} h$ where $C_{2}$ is some small positive integer. It is therefore of interest to study asymptotically the optimal number of iterations required, $n(h)$, as $h$ goes to zero. The concrete optimization results for first and second order transmission conditions are given in the following theorem.

Theorem 5.6 (Minimal number of iterations). To reach a precision $\epsilon$ for propagating waves using an overlap $\delta$, the minimum number of iterations the optimized 
Schwarz waveform relaxation algorithm with first order optimized transmission conditions needs is $\lceil n\rceil$ where $n$ solves

$$
\left(\frac{\sqrt{c T}-\sqrt{n \delta}}{\sqrt{c T}+\sqrt{n \delta}}\right)^{n}=\epsilon
$$

If the precision and overlap are related to the mesh parameter $h, \epsilon=C_{1} h^{p}$ and $\delta=C_{2} h$, then the number of iterations is asymptotically

$$
n(h) \approx\left(\frac{T c}{4 C_{2}}\right)^{1 / 3}\left(\frac{(p \log h)^{2}}{h}\right)^{1 / 3} .
$$

With the second order optimized transmission conditions the minimum number of iterations is $\lceil n\rceil$ with $n$ solving

$$
\left(\frac{\sqrt{c T+n \delta}+\sqrt{n \delta}-\sqrt{c T}-\sqrt{2} \frac{(c T)^{3 / 4}}{(n \delta) 1 / 4}}{+\sqrt{c T+n \delta}+\sqrt{n \delta}-\sqrt{c T}+\sqrt{2} \frac{(c T)^{3 / 4}}{(n \delta)^{1 / 4}}} \cdot \frac{\sqrt{2} \frac{(n \delta)^{3 / 4}}{(T)^{1 / 4}}-\sqrt{c T+n \delta}-\sqrt{n \delta}+\sqrt{c T}}{\sqrt{2} \frac{(n \delta)^{3 / 4}}{(c T)^{1 / 4}}+\sqrt{c T+n \delta}+\sqrt{n \delta}-\sqrt{c T}}\right)^{n}=\epsilon
$$

which gives asymptotically with $\epsilon=C_{1} h^{p}$ and $\delta=C_{2} h$

$$
n(h) \approx\left(\frac{T c}{64 C_{2}}\right)^{1 / 5}\left(\frac{(p \log h)^{4}}{h}\right)^{1 / 5} .
$$

Proof. If we use a first order approximation optimized for the interval $[\underline{\tau}, 1]$, then with the optimal parameter $\tau_{1}=\sqrt{\underline{\tau}}$ from Lemma 5.4 we find for the maximum of the convergence rate

$$
\rho_{o p t 1}=\frac{1-\sqrt{\underline{\tau}}}{1+\sqrt{\underline{\tau}}}
$$

Using $\underline{\tau}=\frac{n \delta}{c T}$, the equation for the optimal number of iterations (5.12) follows from (5.11). Using $\delta=C_{2} h$ and $\epsilon=C_{1} h^{p}$ in equation (5.12), taking the logarithm on both sides and assuming that $n h$ goes to zero, we find, expanding on the left, for the principal term

$$
-2 n\left(\frac{n C_{2}}{c T}\right)^{1 / 2} \sqrt{h} \approx p \log h
$$

which leads to (5.13).

For the second order case, we find with the optimal parameters from Lemma 5.4 for the maximum of the convergence rate

$$
\rho_{\text {opt } 2}=\frac{\sqrt{1+\underline{\tau}}+\sqrt{\underline{\tau}}-1-\sqrt{2}(\underline{\tau})^{-1 / 4}}{\sqrt{1+\underline{\tau}}+\sqrt{\underline{\tau}}-1+\sqrt{2}(\underline{\tau})^{-1 / 4}} \cdot \frac{\sqrt{2}(\underline{\tau})^{3 / 4}-\sqrt{1+\underline{\tau}}-\sqrt{\underline{\tau}}+1}{\sqrt{2}(\underline{\tau})^{3 / 4}+\sqrt{1+\underline{\tau}}+\sqrt{\underline{\tau}}-1}
$$

which leads with $\underline{\tau}=\frac{n \delta}{c T}$ and (5.11) to (5.14). Using $\delta=C_{2} h$ and $\epsilon=C_{1} h^{p}$ in equation (5.14), taking the logarithm on both sides and expanding on the left, assuming again that $n h$ goes to zero, we find after a lengthy calculation for the principal term

$$
-2^{3 / 2} n\left(\frac{n C_{2}}{c T}\right)^{1 / 4} h^{1 / 4} \approx p \log h
$$

which leads to (5.15). 
As expected the number of iterations cannot be independent of $h$ if the overlap depends on $h$, but the dependence is much weaker than for the classical Schwarz waveform relaxation method, where the number of iterations grows like $1 / h$ as seen in Theorem 2.1 if the overlap $\delta=O(h)$. Note also that the second order optimized Schwarz waveform relaxation algorithm has a weaker dependence on $h$ than the first order optimized one.

\section{NumERICAL EXPERIMENTS}

We show two sets of numerical experiments, one to show how the optimized absorbing boundary conditions with a layer can be used to truncate an infinite computational domain and one that shows what the impact of such conditions on the Schwarz algorithm is.

6.1. Truncated computational domains. We pose the wave equation (2.1) on the infinite domain $\Omega=(0, \infty) \times(0,1)$ with homogeneous boundary conditions. We truncate the domain at $x=\beta$ and are interested in the solution on the bounded domain $(0, \alpha) \times(0,1)$ where $\alpha<\beta$ and thus the layer width $\delta=\beta-\alpha$. We chose for our experiment $\alpha=0.46$ and $\beta=0.54$. The wave speed is chosen to be $c=1$ and we simulate in the time interval $t \in[0,1 / 2]$. As the initial condition we use a sharp spike,

$$
u_{0}(x, y)=2 e^{-2000\left((x-0.45)^{2}+(y-0.5)^{2}\right)}, \quad u_{0}^{\prime}(x, y)=0
$$

This initial condition generates a circular wave that expands into all directions as one can see from the three snapshots of the solution at $t=0, t=1 / 4$ and $t=1 / 2$ in Figure 6. We discretize the wave equation with centered finite differences, with discretization parameters $\Delta x=\Delta y=1 / 149$ and $\Delta t=1 / 214$ which is close to the
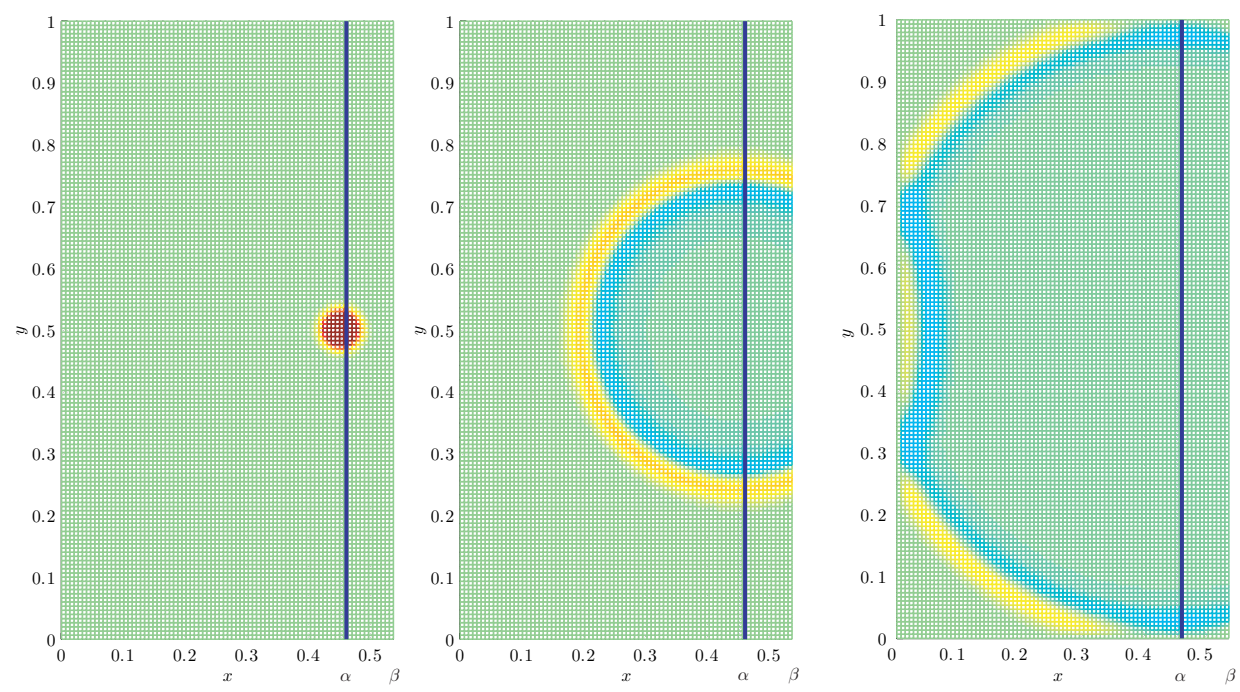

Figure 6. The solution without truncation shown on the truncated computational domain at times $t=0, t=1 / 4$, and $t=1 / 2$. We are interested in the solution on the left of the line $x=\alpha$. 

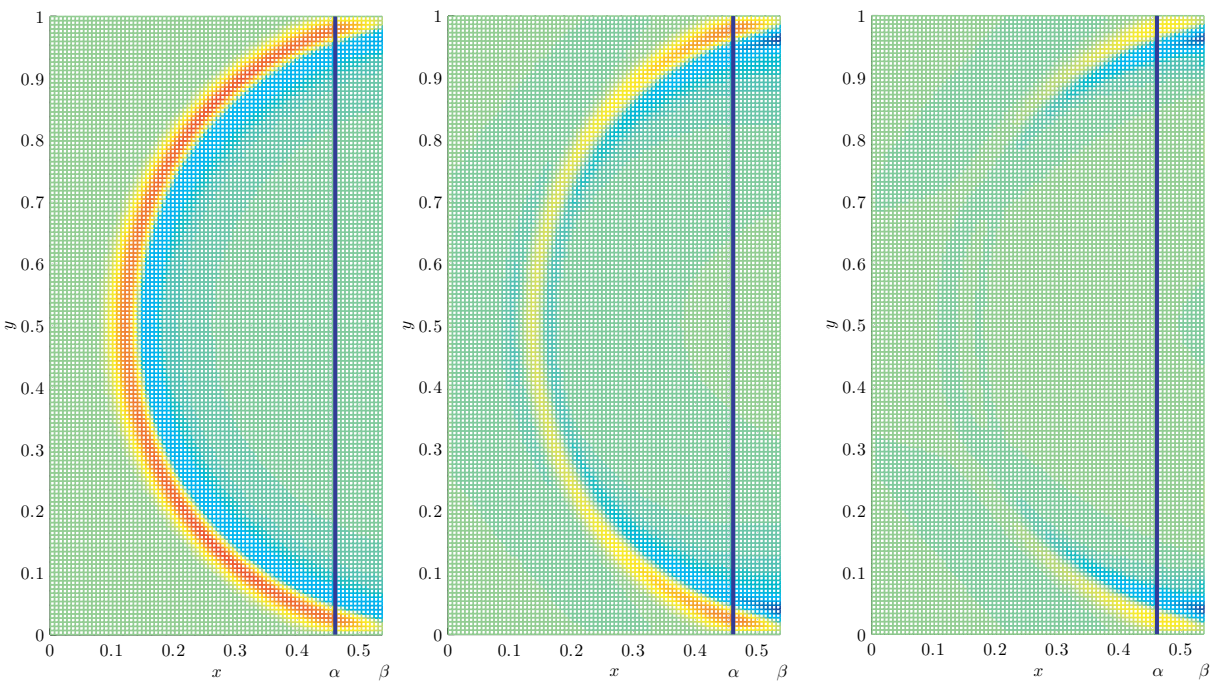

Figure 7. Error caused by the truncation, on the left by Dirichlet, in the middle by a first order, and on the right by a second order orthogonally absorbing condition.

CFL condition of the numerical scheme. To discretize the approximate absorbing boundary conditions, we chose the scheme used in [15. Our reference solution is computed on a bigger domain, and we call this solution the exact solution. In Figure 7 we show, amplified by a factor five compared to Figure 6, the error between the truncated solution and the exact solution on the left for a truncation with Dirichlet condition, in the middle for a truncation with a first order absorbing

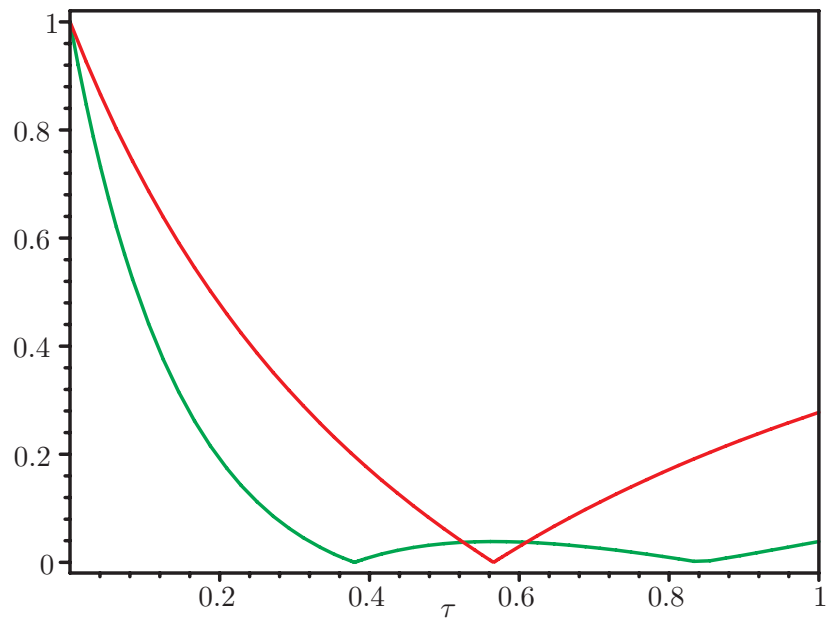

Figure 8. Magnitude of the reflection coefficient as a function of $\tau$ for the first and second order optimized absorbing boundary conditions. 

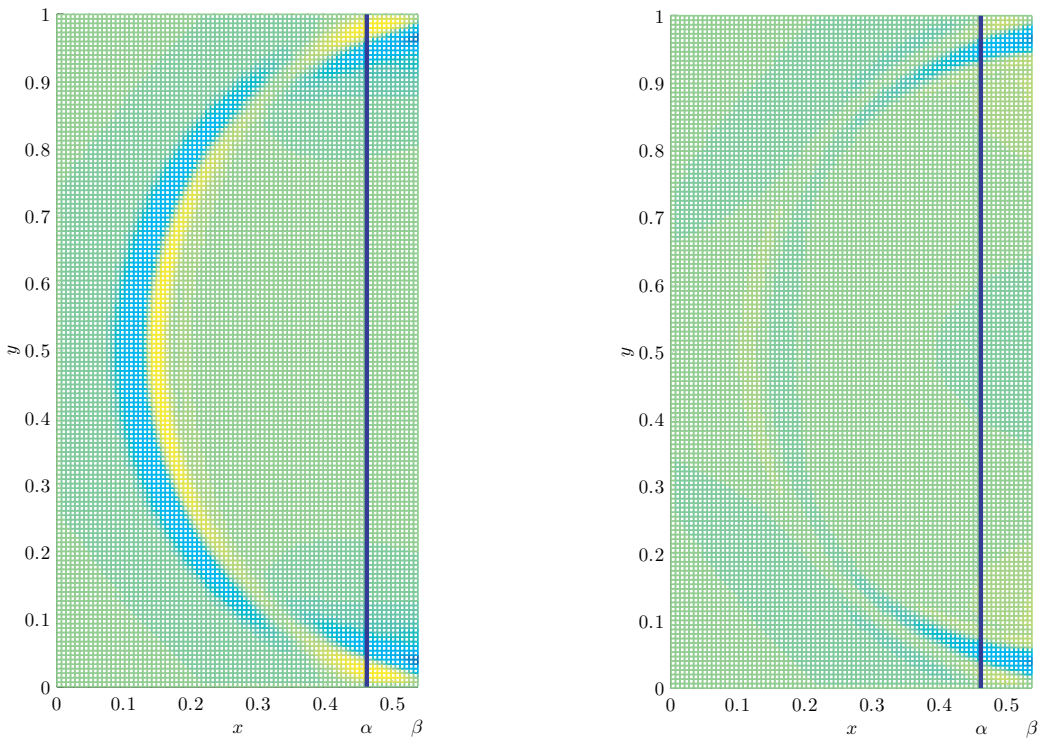

Figure 9. Only a small and balanced error remains when optimized absorbing conditions are used; on the left first order and on the right second order.

boundary condition which exactly absorbs waves that come in orthogonally to the interface, $\tau_{1}=1$, and on the right for a second order absorbing boundary condition for orthogonally incoming waves, $\tau_{1}=\tau_{2}=1$. The absorbing boundary conditions reduce the error considerably, but the error is not reduced uniformly, the oblique part of the wave still carries an important amplitude, as one can see in and close to the layer. This problem is fixed when the optimized absorbing boundary conditions are used. For the problem at hand, we find that $\cos \theta_{\max }=\frac{2 \delta}{c T}=0.32$. The reflection coefficient obtained for the first and second order optimized absorbing boundary conditions are shown in Figure 8 . Note how the optimized rates are equilibrating the maxima over $\tau \in\left[\cos \theta_{\max }, 1\right]$. Figure 9 shows the results obtained with these optimized absorbing boundary conditions. One can see from the numerical experiment that the wave fronts are indeed balanced between the orthogonal wave and the ones close to the boundary of the layer. We finally show in Table 1 the error caused by the truncation measured in the $L^{\infty}$ and $L^{2}$ norms in space at time $t=T$.

TABLE 1. Comparison of the error caused by the truncation of the domain by the various methods.

\begin{tabular}{|c|c|c|c|c|c|}
\hline \multirow{2}{*}{$\begin{array}{c}\text { error } \\
\text { at } t=T\end{array}$} & \multirow{2}{*}{ Dirichlet } & \multicolumn{2}{|c|}{ First Order } & \multicolumn{2}{c|}{ Second Order } \\
\cline { 3 - 6 } & & Orthogonal & Optimized & Orthogonal & Optimized \\
\hline$L^{\infty}$ & $100 \%$ & $42 \%$ & $26 \%$ & $27 \%$ & $18 \%$ \\
\hline$L^{2}$ & $100 \%$ & $22 \%$ & $21 \%$ & $10 \%$ & $5 \%$ \\
\hline
\end{tabular}


6.2. Domain decomposition. We show now convergence results for the optimized Schwarz waveform relaxation algorithm given in (4.1) with $c=1$. We use first as transmission conditions $\mathcal{B}_{i}, i=1,2$, either the classical Dirichlet conditions, the first order orthogonal absorbing conditions, or the second order orthogonal absorbing conditions. Our simulation is performed on the unit square with two subdomains of the same size and overlap 0.08. We use homogeneous boundary conditions and the initial data

$$
u_{0}(x, y)=2 e^{100\left((x-0.25)^{2}+(y-0.5)^{2}\right)}+2 e^{100\left((x-0.75)^{2}+(y-0.5)^{2}\right)}, \quad u_{0}^{\prime}(x, y)=0 .
$$

We start the iteration with the zero initial guess, $u_{1}^{0}=u_{2}^{0}=0$, and simulate in the time interval $t \in[0,1]$. The computations are performed with a centered finite difference scheme with $\Delta x=\Delta y=1 / 99$ and $\Delta t=1 / 141$, so that the discretization is close to the CFL. Figure 10 shows the convergence behavior of the algorithm. One can clearly see that the transmission conditions are of great importance for a good performance of the algorithm. The classical Dirichlet conditions lead to convergence after 15 steps, the number predicted by Theorem 2.1 and the algorithm seems to stagnate before, as expected. The optimized transmission conditions lead to linear convergence at a rate depending on the degree of approximation in the optimized transmission conditions. In practice one is interested in the solution only up to the discretization error, which would mean for the example that about 5 iterations are necessary for the first order and 3 for the second order transmission condition.

Now we assume that we have an a priori tolerance $\epsilon=10^{-2}$, and we use the results of Theorem 5.6. For the first order optimized transmission condition this leads to a number of iterations $n=3.7459 \approx 3-4$ with the parameter $\cos \theta_{\max }=$ 0.2997 which corresponds to an angle of $73^{\circ}$. The optimal parameter to be used is

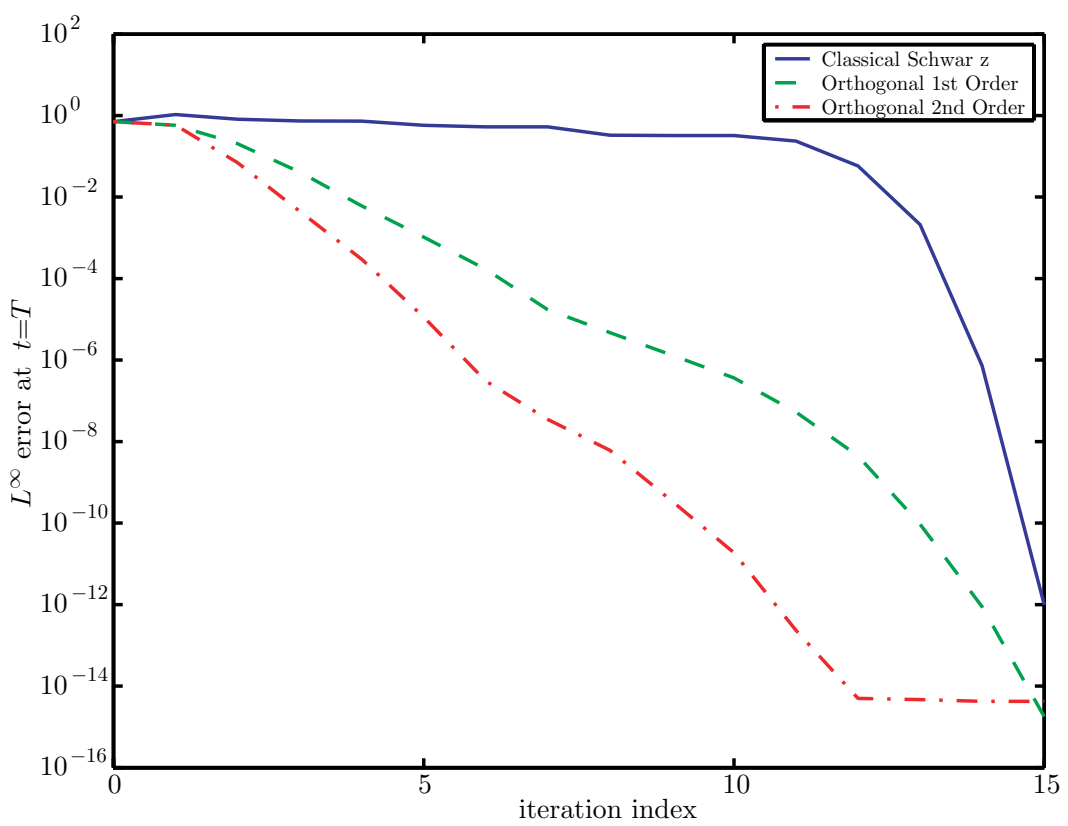

Figure 10. The impact the transmission conditions have on the Schwarz waveform relaxation algorithm. 
TABLE 2. Optimized absorption for an a priori accuracy of $\epsilon=$ $10^{-2}$. The first order optimized method optimized for 3-4 steps, and the second order one for 2 steps.

\begin{tabular}{|r|c|c|c|c|c|c|}
\hline Iteration & 0 & 1 & 2 & 3 & 4 & 5 \\
\hline Dirichlet & 0.7059 & 1.0555 & 0.8146 & 0.7340 & 0.7321 & 0.5760 \\
\hline Orthogonal O1 & 0.7059 & 0.5793 & 0.2035 & 0.0413 & 0.0061 & 0.0010 \\
\hline Optimized O1 & 0.7059 & 0.4403 & 0.1132 & 0.0216 & 0.0062 & 0.0018 \\
\hline Orthogonal O2 & 0.7059 & 0.5853 & 0.0701 & 0.0045 & 0.0003 & 0.0000 \\
\hline Optimized O2 & 0.7059 & 0.5847 & 0.0415 & 0.0099 & 0.0030 & 0.0004 \\
\hline
\end{tabular}

therefore $\tau_{1}=0.5474$. In Table 2 we show the error at the end of the time interval in the $L^{\infty}$ norm. One can see that the method optimized for 3-4 iterations indeed gives a smaller error, by a factor of 2 , at iteration 3 , over the orthogonal absorbing one.

For the second order optimized transmission conditions the optimized number of iterations for a tolerance of $\epsilon=10^{-2}$ is $n=1.9540 \approx 2$ with the parameter $\cos \theta_{\max }=0.1563$ which corresponds to an angle of $81^{\circ}$. The optimal parameters to be used are $\tau_{1}=0.2093$ and $\tau_{2}=0.7469$. Again in Table 2 one can see that at iteration 2 the error is almost a factor 2 smaller than for the orthogonally absorbing method.

Finally one can also see from these numerical experiments that orthogonal absorption is a good option, if one does not have an a priori tolerance $\epsilon$ : in Table 2 at iteration 5 the orthogonal absorbing versions of the algorithm have a smaller error than the optimized ones. This is in accordance with our analysis, since when $\epsilon$ goes to zero, $\theta_{\max }^{n}$ in (5.11) goes to zero and the optimal choice is orthogonal absorption.

\section{Conclusions}

We introduced a new optimization strategy for absorbing boundary conditions for the wave equation. The strategy is motivated by a domain decomposition method with overlap, namely the Schwarz method, and hence includes a small layer which is part of the absorbing boundary condition. The new optimized absorbing boundary conditions can be used to truncate computational domains and for parallel computing, where they greatly enhance the performance of the classical Schwarz waveform relaxation method.

\section{REFERENCES}

1. Alain Bamberger, Roland Glowinski, and Quang H. Tran, A domain decomposition method for the acoustic wave equation with discontinuous coefficients and grid change, SIAM Journal on Numerical Analysis 34 (1997), no. 2, 603-639. MR 98c:65161

2. A. Bayliss and E. Turkel, Radiation boundary conditions for wave-like equations, Comm. Pure and Appl. Math. 33 (1980), no. 6, 707-725. MR 82b:65091

3. J. D. Benamou and B. Després, A domain decomposition method for the Helmholtz equation and related optimal control problems, J. of Comp. Physics 136 (1997), 68-82. MR 98c:65162

4. Morten Bjørhus, On domain decomposition, subdomain iteration and waveform relaxation, Ph.D. thesis, University of Trondheim, Norway, 1995.

5. Xiao-Chuan Cai, Additive Schwarz algorithms for parabolic convection-diffusion equations, Numer. Math. 60 (1991), no. 1, 41-61. MR 93a:65127

6. Multiplicative Schwarz methods for parabolic problems, SIAM J. Sci Comput. 15 (1994), no. 3, 587-603. MR 95c:65178 
7. Xiao-Chuan Cai, Mario A. Casarin, Frank W. Elliott Jr., and Olof B. Widlund, Overlapping Schwarz algorithms for solving Helmholtz's equation, Domain decomposition methods, 10 (Boulder, CO, 1997), Amer. Math. Soc., Providence, RI, 1998, pp. 391-399.

8. Philippe Charton, Frédéric Nataf, and Francois Rogier, Méthode de décomposition de domaine pour l'équation d'advection-diffusion, C. R. Acad. Sci. 313 (1991), no. 9, 623-626. MR 92j:65192

9. Philippe Chevalier and Frédéric Nataf, Symmetrized method with optimized second-order conditions for the Helmholtz equation, Domain decomposition methods, 10 (Boulder, CO, 1997), Amer. Math. Soc., Providence, RI, 1998, pp. 400-407.

10. P. Collino, G. Delbue, P. Joly, and A. Piacentini, A new interface condition in the nonoverlapping domain decomposition for the Maxwell equations Helmholtz equation and related optimal control, Comput. Methods Appl. Mech. Engrg. 148 (1997), 195-207. MR 98f:78001

11. Armel de La Bourdonnaye, Charbel Farhat, Antonini Macedo, Frédéric Magoulès, and François-Xavier Roux, A nonoverlapping domain decomposition method for exterior Helmholtz problems, Domain decomposition methods, 10 (Boulder, CO, 1997) (Providence, RI), Amer. Math. Soc., 1998, pp. 42-66. MR 99f:65190

12. Bruno Després, Patrick Joly, and Jean E. Roberts, A domain decomposition method for the harmonic Maxwell equations, Iterative methods in linear algebra (Brussels, 1991) (Amsterdam), North-Holland, 1992, pp. 475-484.

13. J. Douglas, Jr. and D. B. Meade, Second-order transmission conditions for the Helmholtz equation, Ninth International Conference on Domain Decomposition Methods (P. E. Bjørstad, M. Espedal, and D. Keyes, eds.), ddm.org, 1997, pp. 434-440.

14. B. Engquist and L. Halpern, Long-time behaviour of absorbing boundary conditions, Math. Methods Appl. Sci. 13 (1990), no. 3, 189-203. MR 91m:35045

15. B. Engquist and A. Majda, Absorbing boundary conditions for the numerical simulation of waves, Math. Comp. 31 (1977), no. 139, 629-651. MR 55:9555

16. Martin J. Gander, Overlapping Schwarz for parabolic problems, Ninth International Conference on Domain Decomposition Methods (Petter E. Bjørstad, Magne Espedal, and David Keyes, eds.), ddm.org, 1997, pp. 97-104.

17. - Overlapping Schwarz waveform relaxation for parabolic problems, Tenth International Conference on Domain Decomposition Methods (J. Mandel, C. Farhat, and X.-C. Cai, eds.), AMS, Contemporary Mathematics 218, 1998. MR 99f:65005

18. _ A waveform relaxation algorithm with overlapping splitting for reaction diffusion equations, Numerical Linear Algebra with Applications 6 (1998), 125-145. MR 2000m:65110

19. - Optimized Schwarz methods for Helmholtz problems, Thirteenth international conference on domain decomposition, 2001, pp. 245-252.

20. Martin J. Gander and Laurence Halpern, Méthodes de décomposition de domaines pour l'équation des ondes en dimension 1, C. R. Acad. Sci. Paris I (2001), no. 333, 589-592. MR 2002i:65096

21. Un algorithme discret de décomposition de domaines pour l'équation des ondes en dimension 1, C. R. Acad. Sci. Paris I (2001), no. 333, 699-702. MR 2002i:65097

22. Martin J. Gander, Laurence Halpern, and Frédéric Nataf, Optimal convergence for overlapping and nonoverlapping Schwarz waveform relaxation, Eleventh international Conference of Domain Decomposition Methods (C-H. Lai, P. Bjørstad, M. Cross, and O. Widlund, eds.), ddm.org, 1999.

23. Martin J. Gander, Laurence Halpern, and Frédéric Nataf, Optimized Schwarz methods, Twelfth International Conference on Domain Decomposition Methods, Chiba, Japan (Bergen) (Tony Chan, Takashi Kako, Hideo Kawarada, and Olivier Pironneau, eds.), Domain Decomposition Press, 2001, pp. 15-28.

24. Martin J. Gander, Frédéric Magoulès, and Frédéric Nataf, Optimized Schwarz methods without overlap for the Helmholtz equation, SIAM J. Sci. Comput. 24 (2002), no. 1, 38-60.

25. Martin J. Gander and Andrew M. Stuart, Space time continuous analysis of waveform relaxation for the heat equation, SIAM J. 19 (1998), 2014-2031. MR 99h:65164

26. Martin J. Gander and Hongkai Zhao, Overlapping Schwarz waveform relaxation for parabolic problems in higher dimension, Proceedings of Algoritmy 14 (A. Handlovičová, Magda Komorníkova, and Karol Mikula, eds.), Slovak Technical University, September 1997, pp. 42-51.

27. Eldar Giladi and Herbert Keller, Space time domain decomposition for parabolic problems, Numerische Mathematik 93 (2002), no. 2, 279-313. 
28. Laurence Halpern, Absorbing boundary conditions for the discretization schemes of the onedimensional wave equation, Mathematics of Computation 38 (1982), no. 158, 415-429. MR 83d:65245

29. - Artificial boundary conditions for the advection-diffusion equations, Math. Comp. 174 (1986), 425-438. MR 87e:76005

30. Robert L. Higdon, Initial-boundary value problems for linear hyperbolic systems, SIAM Rev. 28 (1986), no. 2, 177-217. MR 88a:35138

31. Caroline Japhet, Optimized Krylov-Ventcell method. Application to convection-diffusion problems, Proceedings of the 9th international conference on domain decomposition methods (Petter E. Bjørstad, Magne S. Espedal, and David E. Keyes, eds.), ddm.org, 1998, pp. 382-389.

32. Caroline Japhet, Frederic Nataf, and Francois Rogier, The optimized order 2 method. Application to convection-diffusion problems, Future Generation Computer Systems FUTURE 18 (2001).

33. Caroline Japhet, Frederic Nataf, and Francois-Xavier Roux, The Optimized Order 2 Method with a coarse grid preconditioner. Application to convection-diffusion problems, Ninth International Conference on Domain Decomposition Methods in Science and Engineering (P. Bjorstad, M. Espedal, and D. Keyes, eds.), John Wiley \& Sons, 1998, pp. 382-389.

34. Jacques-Louis Lions and Enrico Magenes, Problèmes aux limites non homogènes et applications, Travaux et recherches mathématiques, vol. 17-18, Dunod, 1968. MR 40:512 MR 40:513

35. Lois C. McInnes, Romeo F. Susan-Resigna, David E. Keyes, and Hafiz M. Atassi, Additive Schwarz methods with nonreflecting boundary conditions for the parallel computation of Helmholtz problems, Domain decomposition methods, 10 (Boulder, CO, 1997), Amer. Math. Soc., 1998, pp. 325-333.

36. Gérard A. Meurant, Numerical experiments with a domain decomposition method for parabolic problems on parallel computers, Fourth International Symposium on Domain Decomposition Methods for Partial Differential Equations (Philadelphia, PA) (Roland Glowinski, Yuri A. Kuznetsov, Gérard A. Meurant, Jacques Périaux, and Olof Widlund, eds.), SIAM, 1991. MR 92a:65023

37. Ulla Miekkala and Olavi Nevanlinna, Convergence of dynamic iteration methods for initial value problems, SIAM J. Sci. Stat. Comput. 8 (1987), 459-482. MR 89f:65076

38. Frédéric Nataf, Absorbing boundary conditions in block Gauss-Seidel methods for convection problems, Math. Models Methods Appl. Sci. 6 (1996), no. 4, 481-502. MR 97g:65259

39. Frédéric Nataf and Francis Nier, Convergence rate of some domain decomposition methods for overlapping and nonoverlapping subdomains, Numerische Mathematik 75 (1997), no. 3, 357-77. MR 98m:65219

40. Donald J. Newman, Rational approximation to $|x|$, Michigan Math. J. 11 (1964), 11-14. MR 30:1344

41. Alfio Quarteroni and Alberto Valli, Domain decomposition methods for partial differential equations, Oxford Science Publications, 1999. MR 2002i:65002

42. Andrea Toselli, Some results on overlapping Schwarz methods for the Helmholtz equation employing perfectly matched layers, Tech. Report 765, Courant Institute, New York, June 1998.

43. Loic Tourrette and Laurence Halpern (eds.), Absorbing boundaries and layers, domain decomposition methods, application to large scale computations, Novascience, 2001.

44. Lloyd N. Trefethen and Laurence Halpern, Well-posedness of one-way wave equations and absorbing boundary conditions, Math. of Comp. 47 (1986), no. 167, 421-435. MR 88b:65148

45. Eugene L. Wachspress, Optimum alternating-direction-implicit iteration parameters for a model problem, J. Soc. Indust. Appl. Math. 10 (1962), 339-350. MR 27:921

46. Yunhai Wu, Xiao-Chuan Cai, and David E. Keyes, Additive Schwarz methods for hyperbolic equations, Tenth International Conference on Domain Decomposition Methods (J. Mandel, C. Farhat, and X.-C. Cai, eds.), AMS, Contemporary Mathematics 218, 1998, pp. 513-521.

Department of Mathematics and Statistics, McGill University, Montreal, Canada Département de Mathématiques, Université Paris XiII, 93430 Villetaneuse, France E-mail address: halpern@math.univ-paris13.fr 